\title{
Time-dependent density functional theory: Derivation of gradient-corrected dynamical exchange-correlational potentials
}

\author{
Jianmin Tao* and Giovanni Vignale \\ Department of Physics, University of Missouri-Columbia, Columbia, Missouri 65211, USA \\ I. V. Tokatly \\ Lehrstuhl für Theoretische Festkörperphysik, Universität Erlangen-Nürnberg, Staudtstrasse 7/B2, 91058 Erlangen, Germany \\ and Moscow Institute of Electronic Technology, Zelenograd, 124498 Russia
}

(Received 11 April 2007; revised manuscript received 16 August 2007; published 30 November 2007)

\begin{abstract}
We have recently proposed an approximation for the dynamical exchange-correlation (XC) potentials of time-dependent current-density functional theory beyond the local density approximation [Phys. Rev. Lett. 97, 036403 (2006)]. The novel feature of the approximation is that the dependence of the dynamical XC potentials upon the density gradient and other inhomogeneity parameters (e.g., the Laplacian of the density and the kinetic energy density) is introduced by applying the generalized gradient approximation (GGA) and metaGGA to the calculation of the XC stress tensor. The scheme may allow a more accurate treatment of the dynamical properties of an inhomogeneous system, while reducing to the exact form for slowly varying densities and slowly varying external potentials. In this work, we present in detail the derivation of this XC potential, spell out the underlying assumptions, and explain their physical basis.
\end{abstract}

DOI: 10.1103/PhysRevB.76.195126

PACS number(s): 71.15.Mb, 31.15.Ew, 71.45.Gm

\section{INTRODUCTION}

The popularity of time-dependent density functional theory $^{1,2}$ (TDDFT) for electronic structure calculations has been dramatically growing in recent years. Formally it is an exact many-body theory. In practice, the dynamical exchange-correlation (XC) potential must be approximated. The simplest approximation is the adiabatic approximation, ${ }^{3}$ which makes use of the ground-state XC potential but replaces the ground-state density $n_{0}(\mathbf{r})$ with the instantaneous density $n(\mathbf{r}, t)$-namely,

$$
V_{\mathrm{xc}}^{a d}([n] ; \mathbf{r}, t)=\left.\frac{\delta\left[n_{0}(\mathbf{r}) \epsilon_{\mathrm{xc}}\left(\left[n_{0}\right] ; \mathbf{r}\right)\right]}{\delta n_{0}(\mathbf{r})}\right|_{n_{0}(\mathbf{r})=n(\mathbf{r}, t)},
$$

where $\epsilon_{\mathrm{xc}}$ is the equilibrium $\mathrm{XC}$ energy per electron. This approximation-which openly ignores the history of the system prior to time $t$-has been widely used to calculate the single-particle low-lying excitation energies ${ }^{4,5}$ and van der Waals coefficients ${ }^{6}$ of molecules and solids. However, it has several well-known limitations, both qualitative and quantitative. On the qualitative side, it does not allow for dissipation $^{7,8}$ and fails completely for multiparticle excitations $s^{9,10}$ or charge transfer processes ${ }^{11-13}$ or similar problems ${ }^{14}$ for excitations in spatially separated systems that do not involve charge transfer. On the quantitative side, it significantly overestimates ${ }^{15-18}$ the dielectric polarizability of insulators and in many cases underestimates the excitation energies. $^{19}$

The first attempt to go beyond the adiabatic local density approximation (ALDA) was made by Gross and Kohn ${ }^{20}$ (GK). They proposed a time- or frequency-dependent linearorder correction to the ALDA. This, however, was later found $^{21,22}$ to violate the "harmonic potential theorem" and other exact conditions. After a careful analysis of this difficulty, Vignale and $\mathrm{Kohn}^{23}$ (VK) concluded that (i) the dy- namical XC potential is an "ultranonlocal" functional of the density, and thus a consistent local density approximation does not exist; (ii) a local density approximation is still possible if the current density $\mathbf{j}(\mathbf{r}, t)$ rather than the electron density $n(\mathbf{r}, t)$ is chosen as the basic variable. Based on these observations, VK introduced the time-dependent currentdensity functional theory. ${ }^{23}$ In practice, they derived an explicit frequency-dependent expression, within linear response theory, for the XC vector potential $\mathbf{A}_{\mathrm{xc}}(\mathbf{r}, t)$ and the associated XC electric field $\mathbf{E}_{\mathrm{xc}}(\mathbf{r}, t)=-(1 / c) \partial \mathbf{A}_{\mathrm{xc}}(\mathbf{r}, t) / \partial t$ (where $c$ is the speed of light) of a system of slowly varying density (characterized by a local wave vector $q=\left|\nabla n_{0}\right| / n_{0}$ ) and slowly varying external potential (characterized by wave vector $k$ ).

In this theory the Fourier transform in time of the induced $\mathrm{XC}$ electric field $\mathbf{E}_{\mathrm{xc}}(\mathbf{r}, \omega)=(i \omega / c) \mathbf{A}_{\mathrm{xc}}(\mathbf{r}, \omega)$ (where $\omega$ is the angular frequency of the external perturbing field) is given by 24

$$
e E_{\mathrm{xc}, \mu}(\mathbf{r}, \omega)=\partial_{\mu} V_{\mathrm{xc}}^{\mathrm{ALDA}}(\mathbf{r}, \omega)+\frac{1}{n_{0}(\mathbf{r})} \partial_{\nu} \Delta P_{\mu \nu}^{\mathrm{h}, \mathrm{xc}}(\mathbf{r}, \omega),
$$

where $e$ is the absolute value of the electron charge, $\partial_{\mu}$ $=\partial / \partial r_{\mu}$, and $\mu, \nu$ are Cartesian indices with the convention that repeated indices are summed over. The first term on the right-hand side of Eq. (2) is the ALDA part, and the second is the frequency-dependent dynamical correction. The quantity $\Delta P_{\mu \nu}^{\mathrm{h}, \mathrm{xc}}$ is the dynamical $\mathrm{XC}$ stress tensor of the homogeneous (h) electron liquid, evaluated at the local density:

$$
\begin{aligned}
\Delta P_{\mu \nu}^{\mathrm{h}, \mathrm{xc}}= & \mu_{\mathrm{xc}}^{\mathrm{h}}\left(n_{0}, \omega\right)\left(u_{\mu \nu}-\frac{\delta_{\mu \nu}}{3} u_{\alpha \alpha}\right) \\
& +\frac{\delta_{\mu \nu}}{2}\left[K_{\mathrm{xc}}^{\mathrm{h}}\left(n_{0}, \omega\right)-K_{\mathrm{xc}}^{\mathrm{h}, 0}\left(n_{0}\right)\right] u_{\alpha \alpha},
\end{aligned}
$$

the divergence of which gives the linear-order correction to 
the ALDA. Here $u_{\mu \nu}$ is the negative of the strain tensor defined as

$$
u_{\mu \nu} \equiv-\partial_{\nu} u_{\mu}-\partial_{\mu} u_{\nu}
$$

where $u_{\mu}(\mathbf{r}, \omega)=-j_{\mu}(\mathbf{r}, \omega) /\left(i \omega n_{0}\right)$ is the Fourier transform in time of the elastic displacement field. In Eq. (3), $K_{\mathrm{xc}}^{\mathrm{h}}\left(n_{0}, \omega\right)$ and $\mu_{\mathrm{xc}}^{\mathrm{h}}\left(n_{0}, \omega\right)$ are the XC parts of the bulk modulus and shear modulus, respectively, ${ }^{2}$ and

$$
K_{\mathrm{xc}}^{\mathrm{h}, 0}\left(n_{0}\right)=n_{0}^{2} \partial^{2}\left(n_{0} \epsilon_{\mathrm{xc}}\right) / \partial n_{0}^{2}
$$

is the low-frequency $(\omega \rightarrow 0)$ limit of the bulk modulus. The nonadiabatic part of Eq. (2) includes dissipation and retardation effects, and allows in principle for multiparticle excitations. The dissipation arises physically from the production of multiparticle excitations and mathematically from the presence of a component of the effective electric field in phase with the current.

The expressions (2) and (3) for the XC potential were originally derived under the assumption that $q, k \ll k_{\mathrm{F}}, \omega / v_{\mathrm{F}}$, where $k_{F}$ is the local Fermi wave vector and $v_{\mathrm{F}}$ is the Fermi velocity. That is, both the density and the external perturbing potential are slowly varying on the length scales of $k_{\mathrm{F}}^{-1}(\sim$ interelectron distance $)$ and $v_{\mathrm{F}} / \omega(\sim$ distance traveled by an electron during a period of the perturbing field). In practice, the theory has been applied with some success to situations that do not strictly satisfy these conditions, ${ }^{15,16,25-27}$ leading in some cases to considerable improvement upon the ALDA. However, difficulties persist in those cases in which the condition $q \ll k_{\mathrm{F}}, \omega / v_{\mathrm{F}}$ is severely violated-for example, in the calculation of atomic transition energies ${ }^{28}$ and in all situations involving well-localized orbitals.

Recently we have proposed ${ }^{18}$ a way to construct the dynamical XC potential for time-dependent current-density functional theory beyond the local density approximation. This theory is valid for small and slowly varying strain tensor $u_{\alpha \beta}$, but does not assume slow variation of the groundstate density. In this theory, the XC field has the same form of $\mathrm{VK}$, that is,

$$
e E_{\mathrm{xc}, \mu}(\mathbf{r}, \omega)=\partial_{\mu} V_{\mathrm{xc}}^{\mathrm{ad}}(\mathbf{r}, \omega)+\frac{1}{n_{0}(\mathbf{r})} \partial_{\nu} \Delta P_{\mu \nu}^{\mathrm{xc}}(\mathbf{r}, \omega),
$$

but now, at variance with Eq. (2), $\Delta P_{\mu \nu}^{\mathrm{xc}}(\mathbf{r}, \omega)$ is the dynamical XC stress tensor of the inhomogeneous electron liquid. As we shall see, in the low-frequency limit, the vector potential part of the XC electric field $E_{\mathrm{xc}, \mu}$ [the second term of Eq. (2)] does not vanish, so the dynamical XC electric field does not reduce to the static $\mathrm{XC}$ electric field. The relevancy of the XC electric field in the context of static DFT has been discussed earlier by Harbola and Sahni, ${ }^{29}$ Wang and coauthors, ${ }^{30}$ and Holas and March. ${ }^{31}$ Under an approximation of local isotropy, which will be fully described in the following sections, this stress tensor is still connected to the strain tensor by the local relation (3), involving only two elastic constants $K_{\mathrm{xc}}$ and $\mu_{\mathrm{xc}}$ which are now positiondependent functionals of the ground-state density. In particular, we have found that the high-frequency $(\omega \rightarrow \infty)$ limits of the bulk modulus and the shear modulus, denoted by $K_{\mathrm{xc}}^{\infty}$ and $\mu_{\mathrm{xc}}^{\infty}$, respectively, can be expressed as

$$
\begin{aligned}
& K_{\mathrm{xc}}^{\infty}\left(\left[n_{0}\right] ; \mathbf{r}\right)=\frac{10}{9} T_{\mathrm{c}}\left(\left[n_{0}\right] ; \mathbf{r}\right)+\frac{4}{9} W_{\mathrm{xc}}\left(\left[n_{0}\right] ; \mathbf{r}\right), \\
& \mu_{\mathrm{xc}}^{\infty}\left(\left[n_{0}\right] ; \mathbf{r}\right)=\frac{2}{3} T_{\mathrm{c}}\left(\left[n_{0}\right] ; \mathbf{r}\right)-\frac{2}{15} W_{\mathrm{xc}}\left(\left[n_{0}\right] ; \mathbf{r}\right),
\end{aligned}
$$

where $T_{\mathrm{c}}\left(\left[n_{0}\right] ; \mathbf{r}\right)=T\left(\left[n_{0}\right] ; \mathbf{r}\right)-T^{\mathrm{S}}\left(\left[n_{0}\right] ; \mathbf{r}\right)$ is the correlation part of the kinetic energy density and $W_{\mathrm{xc}}\left(\left[n_{0}\right] ; \mathbf{r}\right)$ is the XC part of the potential energy density. Here $T\left(\left[n_{0}\right] ; \mathbf{r}\right)$ and $T^{\mathrm{S}}\left(\left[n_{0}\right] ; \mathbf{r}\right)$ are the kinetic energy densities of the interacting and noninteracting systems, respectively. The kinetic correlation and the $\mathrm{XC}$ potential energy-density functionals can be obtained, for example, from the generalized gradient approximation $^{32,33}$ (GGA) or from the meta-GGA, ${ }^{34}$ or from their hybrids, as we shall discuss in Sec. V C. By making the viscoelastic moduli explicitly dependent on parameters that measure the inhomogeneity of the ground-state density (e.g., the gradient of the density, the orbital kinetic energy density, or the Laplacian of the density) we hope to reduce the difficulty that VK encounter when applied to systems that have strongly inhomogeneous densities (e.g., atoms and molecules) and are exposed to low-frequency fields (e.g., in molecular transport problems ${ }^{35}$ and low-lying excitations of molecules ${ }^{4}$ ). As discussed in Secs. V and VI, the inhomogeneity parameters may improve the description not only for the infinite-frequency part [Eq. (55)] (essentially by the improvement of the ground-state description) but also for the finite-frequency part [Eq. (62)] of the tensor of elasticity through our interpolation formulas [Eq. (71)].

In this paper, we present a detailed derivation of the theory first outlined in Ref. 18. The derivation makes use of the geometric formulation of TDDFT developed by Tokatly. ${ }^{36}$ In the next section we will briefly review the main points of that formulation and show how to calculate the stress tensor of an inhomogeneous system in the comoving (Lagrangian) reference frame. In Sec. III we review the linear response formulation of the problem in terms of the tensor of elasticity and show that the latter naturally separates into frequency-independent and frequency-dependent parts. In Sec. IV we define the exchange-correlation stress tensor and the associated tensor of elasticity. In Sec. V we calculate the high-frequency limit of the XC stress tensor in the laboratory frame. This calculation leads to Eq. (7). First we introduce three approximations to express the stress tensor in terms of the ground-state kinetic and potential energy densities of exchange correlation. Then we subtract from it the part that pertains to the adiabatic approximation. Finally we show how to calculate the kinetic and potential energy densities of exchange and correlation from the static DFT. In Sec. VI, we discuss the frequency dependence of the stress tensor and apply to the present version of the theory the standard interpolation scheme between high- and lowfrequency limits. The low-frequency limit of the bulk modulus within the GGA is calculated in the Appendix.

\section{TIME-DEPENDENT DEFORMATION FUNCTIONAL THEORY OF THE STRESS TENSOR}

One of us (I.V.T.) (see also Refs. 36-39) has recently developed a geometric formulation of TDDFT and called it 
"time-dependent deformation functional theory" (TDDefFT). The main idea is to consider dynamics from the point of view of a local observer moving with a flow of the electron fluid. In this noninertial reference frame the stress tensor $\widetilde{P}_{\mu \nu}$ [see Eq. (19) for definition] as well as any other observable is a universal functional of the deformation tensor $g_{\mu \nu}$ and of the ground-state density $n_{0}$ provided the system evolves from the ground state. In the linear response regime, as we will show below, the deformation tensor is easily expressed in terms of the current density and TDDefFT effectively becomes a time-dependent current-density functional theory.

In order to calculate the universal functional $\widetilde{P}_{\mu \nu}\left[n_{0}, g_{\mu \nu}\right]$ one needs to go to the above mentioned "comoving frame"i.e., a reference frame that moves with the local velocity $\mathbf{v}(\mathbf{r}, t)$ of the electron liquid and in which, therefore, the particle density is time independent and the expectation value of the current is zero everywhere. The transformation to the comoving frame allows us to separate the "trivial" kinematical effects associated with the translation and rotation of a small volume element of the fluid, from the more challenging dynamical effects arising from the deformation of the volume element. Even though the density remains constant, the Hamiltonian is still time dependent, since it contains the time-dependent deformation tensor as well as a fictitious time-dependent vector potential describing inertial forces in the accelerated frame (see details below). The apparent paradox of the situation disappears once it is understood that the inertial forces generated by the vector potential exactly balance the forces created by the deformation tensor, ensuring the vanishing of the current and hence, by virtue of the continuity equation, the constancy of the density. The Hamiltonian, however, does not explicitly involve the timedependent part of the physical external potential: this potential enters the problem only implicitly through the deformation tensor, which it generates. In fact, the dynamics is completely controlled by the deformation tensor and the condition of zero current density. In this sense the many-body problem in the comoving frame is universal: that is, for a given ground-state density $n_{0}(\mathbf{r})$ any observable (e.g., the stress tensor) depends only on $g_{\mu \nu}(\mathbf{r}, t)$, not on the timedependent external potential that gives rise to it.

Let us summarize the main steps in the formulation of the "universal many-body problem" for the stress tensor.

For a given velocity field $\mathbf{v}(\mathbf{r}, t)$ - defined as the expectation value of the current density divided by the expectation value of the density operator-the nonlinear transformation function $\mathbf{r}(\xi, t)$ mapping the "old" coordinates $\mathbf{r}$ to "new" coordinates $\boldsymbol{\xi}$ is defined as the solution of the Cauchy problem

$$
\dot{\mathbf{r}}(\boldsymbol{\xi}, t) \equiv \mathbf{v}(\mathbf{r}(\xi, t), t), \quad \mathbf{r}(\xi, 0) \equiv \boldsymbol{\xi} .
$$

Physically the transformation function $\mathbf{r}(\boldsymbol{\xi}, t)$ is the trajectory of an infinitesimal fluid element, while the "new" independent coordinate $\boldsymbol{\xi}$ is the position of that element at the initial time $t=0$. A local observer moving along this trajectory sees a stationary particle density distribution and zero current density. In the classical continuum mechanics the transformation of coordinates $\mathbf{r} \rightarrow \boldsymbol{\xi}$ is commonly referred to as the transformation from Eulerian to Lagrangian formulations of the theory. The time-dependent transformation of coordinates $\mathbf{r} \rightarrow \boldsymbol{\xi}$ induces a change of metric $(d \mathbf{r})^{2}=g_{\mu \nu} d \xi_{\mu} d \xi_{\nu}$, where $g_{\mu \nu}(\xi, t)$ is the Green's deformation tensor

$$
g_{\mu \nu}=\frac{\partial r_{\alpha}}{\partial \xi_{\mu}} \frac{\partial r_{\alpha}}{\partial \xi_{\nu}} .
$$

It is convenient to introduce the "displacement field" $\mathbf{u}(\boldsymbol{\xi}, t)$ in the following manner. Integrating both sides of Eq. (8) over $t$, we immediately obtain

$$
\mathbf{r}(t)=\boldsymbol{\xi}+\mathbf{u}(\boldsymbol{\xi}, t),
$$

where

$$
\mathbf{u}(\boldsymbol{\xi}, t)=\int_{0}^{t} d t^{\prime} \mathbf{v}\left(\boldsymbol{\xi}, t^{\prime}\right)
$$

is the displacement of a fluid element from its initial position $\boldsymbol{\xi}$. Notice that to first order in $\mathbf{u}$ the deformation tensor contains the same information as the strain tensor, i.e.,

$$
g_{\mu \nu}=\delta_{\mu \nu}-u_{\mu \nu}
$$

[see Eq. (4)], and the time derivative of $\mathbf{u}(\mathbf{r}, t)$ is simply the current density divided by the ground-state density. In the linear response regime therefore the deformation functional theory reduces to current-density functional theory.

The "universal many-body problem" for the stress tensor in the comoving frame requires that we solve the timedependent Schrödinger equation for the many-body wave function $|\Phi(t)\rangle$ in the comoving frame,

$$
i \partial_{t}|\Phi(t)\rangle=\hat{\tilde{H}}|\Phi(t)\rangle,
$$

with the Hamiltonian

$$
\hat{\tilde{H}}=\hat{\widetilde{T}}+\hat{\tilde{W}}+\hat{V}_{0},
$$

which includes the kinetic energy

$$
\hat{\tilde{T}}=\int d \boldsymbol{\xi} \frac{\hat{\psi}^{\dagger}(\boldsymbol{\xi})}{g^{1 / 4}}\left(i \partial_{\mu}+\frac{e}{c} \mathcal{A}_{\mu}\right) \frac{\sqrt{g} g^{\mu \nu}}{2 m}\left(i \partial_{\nu}+\frac{e}{c} \mathcal{A}_{\nu}\right) \frac{\hat{\psi}(\boldsymbol{\xi})}{g^{1 / 4}},
$$

the interaction potential energy

$$
\hat{\tilde{W}}=\frac{1}{2} \int d \boldsymbol{\xi} \int d \xi^{\prime} w\left(l_{\xi, \xi^{\prime}}\right) \hat{\psi}^{\dagger}(\boldsymbol{\xi}) \hat{\psi}^{\dagger}\left(\boldsymbol{\xi}^{\prime}\right) \hat{\psi}\left(\boldsymbol{\xi}^{\prime}\right) \hat{\psi}(\boldsymbol{\xi})
$$

$\left[w\left(l_{\xi, \xi^{\prime}}\right)=e^{2} / l_{\xi, \xi^{\prime}}\right.$ is the Coulomb interaction potential], and the potential energy of interaction with a static potential $V_{0}(\boldsymbol{\xi})$ [responsible for the ground-state density $\widetilde{n}_{0}(\boldsymbol{\xi})$,

$$
\hat{V}_{0}=\int d \boldsymbol{\xi} V_{0}(\boldsymbol{\xi}) \hat{\psi} \hat{\psi}^{\dagger}(\boldsymbol{\xi}) \hat{\psi}(\boldsymbol{\xi}),
$$

but no time-dependent external potential. Here $V_{0}(\boldsymbol{\xi})$ is the ground-state external potential in the laboratory frame with $\mathbf{r}$ replaced by $\boldsymbol{\xi}, \hat{\psi}(\boldsymbol{\xi})$ and $\hat{\psi}^{\dagger}(\boldsymbol{\xi})$ are the field operators that satisfy the usual fermionic commutation relations, and $l_{\xi, \xi^{\prime}}$ is the length of geodesic connecting points $\boldsymbol{\xi}$ and $\boldsymbol{\xi}^{\prime}$ in the 
space with metric $g_{\mu \nu}(\boldsymbol{\xi}, t)$ [for slowly varying metric $l_{\xi, \xi^{\prime}}$ is explicitly defined immediately below Eq. (23)]. In Eq. (14) the tensor $g^{\mu \nu}$ is the inverse of $g_{\mu \nu}$, and $g$ is the determinant of $g_{\mu \nu}$. The Green's deformation tensor enters the Hamiltonian in Eq. (12) as an effective metric. A crucial feature of the Hamiltonian is the presence of the effective vector potential $\mathcal{A}$, which is determined from the self-consistency equation

$$
\frac{e}{c} \mathcal{A}_{\mu}(\boldsymbol{\xi}, t)=-\frac{i}{2 \widetilde{n}_{0}}\left\langle\Phi(t)\left|\hat{\psi}^{\dagger}(\boldsymbol{\xi}) \partial_{\mu} \hat{\psi}(\boldsymbol{\xi})-\left[\partial_{\nu} \hat{\psi}^{\dagger}(\boldsymbol{\xi})\right] \hat{\psi}(\boldsymbol{\xi})\right| \Phi(t)\right\rangle .
$$

Here $\widetilde{n}_{0}(\boldsymbol{\xi})$ is the particle density in the comoving frame. By construction $\widetilde{n}_{0}(\xi)$ is independent of time and equal to the initial ground-state density $n_{0}(\boldsymbol{\xi})$ :

$$
\widetilde{n}_{0}(\boldsymbol{\xi})=\left\langle\Phi(t)\left|\hat{\psi}^{\dagger}(\boldsymbol{\xi}) \hat{\psi}(\boldsymbol{\xi})\right| \Phi(t)\right\rangle=\left\langle\Phi(0)\left|\hat{\psi}^{\dagger}(\boldsymbol{\xi}) \hat{\psi}(\boldsymbol{\xi})\right| \Phi(0)\right\rangle .
$$

Physically the vector potential $\mathcal{A}(\boldsymbol{\xi}, t)$ describes an inertial force that cancels the stress forces on every fluid element and thus ensures the vanishing of the current density at every point. Indeed, Eq. (17) shows that the diamagnetic part of the current density [the left-hand side of Eq. (17)] exactly cancels the paramagnetic part [the right-hand side of Eq. (17)], causing the net current density to vanish.

The self-consistent system of equations (12)-(17), supplemented with the initial condition $|\Phi(0)\rangle$ (the ground state), uniquely determines the many-body wave function $|\Phi(t)\rangle$ as a universal functional of $\widetilde{n}_{0}$ and $g_{\mu \nu}$. From the knowledge of the wave function one can calculate the stress tensor $\widetilde{P}_{\mu \nu}(\boldsymbol{\xi}, t)$ as follows:

$$
\widetilde{P}_{\mu \nu}\left[g_{\mu \nu}, n_{0}\right](\xi, t)=\left\langle\Phi(t)\left|\hat{\tilde{P}}_{\mu \nu}(\boldsymbol{\xi})\right| \Phi(t)\right\rangle,
$$

where $\hat{\widetilde{P}}_{\mu \nu}(\xi)$ is the stress tensor operator in the comoving frame,

$$
\hat{\widetilde{P}}_{\mu \nu}(\boldsymbol{\xi})=\hat{\widetilde{T}}_{\mu \nu}(\boldsymbol{\xi})+\hat{\widetilde{W}}_{\mu \nu}(\boldsymbol{\xi})
$$

The kinetic and potential parts of the operator $\hat{\widetilde{P}}_{\mu \nu}(\xi)$ are defined via variational derivatives of the corresponding terms in the Hamiltonian [see Eqs. (14) and (15)]:

$$
\hat{\widetilde{T}}_{\mu \nu}(\boldsymbol{\xi})=\frac{2}{\sqrt{g}} \frac{\delta \hat{\tilde{T}}}{\delta g^{\mu \nu}}, \quad \hat{\widetilde{W}}_{\mu \nu}(\boldsymbol{\xi})=\frac{2}{\sqrt{g}} \frac{\delta \hat{\tilde{W}}}{\delta g^{\mu \nu}} .
$$

General representations of $\hat{\widetilde{T}}_{\mu \nu}(\boldsymbol{\xi})$ and $\hat{\tilde{W}}_{\mu \nu}(\boldsymbol{\xi})$ can be found in Ref. 36. These complicated expressions may be dramatically simplified by assuming that the deformation tensor is slowly varying so that its spatial derivative can be neglected. In this case, the expectation value of the kinetic part is given by

$$
\tilde{T}_{\mu \nu}=\frac{1}{m \sqrt{g}}\left[\left.\partial_{\mu} \partial_{\nu^{\prime}} \widetilde{\rho}_{1}\left(\xi, \xi^{\prime}, t\right)\right|_{\xi^{\prime}=\xi}-\frac{g_{\mu \nu}}{4} g^{\alpha \beta} \partial_{\alpha} \partial_{\beta} \tilde{n}(\boldsymbol{\xi})\right],
$$

where $\widetilde{\rho}_{1}\left(\boldsymbol{\xi}, \boldsymbol{\xi}^{\prime}, t\right)=\left\langle\psi^{\dagger}(\boldsymbol{\xi}) \psi\left(\boldsymbol{\xi}^{\prime}\right)\right\rangle$ is the single-particle density matrix, and the expectation value of the potential part is given by

$$
\widetilde{W}_{\mu \nu}(\boldsymbol{\xi}, t)=-\frac{g_{\mu \alpha} g_{\nu \beta}}{2 \sqrt{g}} \int d \boldsymbol{\xi}^{\prime} \frac{\xi_{\alpha}^{\prime} \xi_{\beta}^{\prime}}{\left\|\boldsymbol{\xi}^{\prime}\right\|} \frac{\partial w\left(\left\|\boldsymbol{\xi}^{\prime}\right\|\right)}{\partial\left\|\boldsymbol{\xi}^{\prime}\right\|} \widetilde{\boldsymbol{\rho}}_{2}\left(\boldsymbol{\xi}, \boldsymbol{\xi}^{\prime}, t\right) .
$$

Here $\left\|\boldsymbol{\xi}-\boldsymbol{\xi}^{\prime}\right\|=\sqrt{g_{\mu \nu}\left(\xi_{\mu}-\xi_{\mu}^{\prime}\right)\left(\xi_{\nu}-\xi_{\nu}^{\prime}\right)}$ is the length of geodesic in the limit of homogeneous metric,

$$
\widetilde{\rho}_{2}\left(\boldsymbol{\xi}, \boldsymbol{\xi}^{\prime}, t\right)=\int_{0}^{1} d \lambda \widetilde{\rho}_{2}\left(\boldsymbol{\xi}-(1-\lambda) \boldsymbol{\xi}^{\prime}, \boldsymbol{\xi}+\lambda \boldsymbol{\xi}^{\prime}, t\right),
$$

and $\widetilde{\rho}_{2}\left(\boldsymbol{\xi}, \boldsymbol{\xi}^{\prime}, t\right)=\left\langle\psi^{\dagger}(\boldsymbol{\xi}) \psi^{\dagger}\left(\boldsymbol{\xi}^{\prime}\right) \psi\left(\boldsymbol{\xi}^{\prime}\right) \psi(\boldsymbol{\xi})\right\rangle$. Note the change of notation for $\tilde{\rho}$ to $\overline{\tilde{\rho}}$.

In the kinetic stress tensor (22) we have disregarded the terms with vector potential $\mathcal{A}$ since they do not contribute to the stress tensor of Eq. (19) in the linear response regime (the expectation value of the current density is zero in the ground state). However, such terms should be included in general.

Equations (12)-(21) allow us in principle to calculate the stress tensor $\widetilde{P}_{\mu \nu}(\mathbf{r}, t)$ in the comoving frame. The stress tensor in the laboratory frame will be finally obtained from the transformation

$$
P_{\mu \nu}(\mathbf{r}, t)=\frac{\partial \xi_{\alpha}}{\partial r_{\mu}} \frac{\partial \xi_{\beta}}{\partial r_{\nu}} \widetilde{P}_{\alpha \beta}(\boldsymbol{\xi}(\mathbf{r}, t), t),
$$

where $\boldsymbol{\xi}(\mathbf{r}, t)$ is the inverse of the function $\mathbf{r}(\boldsymbol{\xi}, t)$. In the next section we will show how this problem simplifies in the linear response regime.

\section{LINEAR RESPONSE FORMULATION}

In the linear response the deformation tensor slightly deviates from the $\delta$ symbol and is given by Eq. (11). To first order in the displacement it makes no difference whether the $\mathbf{u}$ is considered as a function of $\mathbf{r}$ or $\boldsymbol{\xi}$.

Expanding the Hamiltonian (13) to first order in the displacement field, we arrive at a linearized Hamiltonian $\hat{\widetilde{H}}_{l}$ of the form

$$
\hat{\tilde{H}}_{l}(t)=\hat{H}+\frac{1}{2} \int d \boldsymbol{\xi} \hat{P}_{\mu \nu}(\boldsymbol{\xi}) u_{\mu \nu}(\boldsymbol{\xi}, t)+\frac{e}{c} \int d \hat{\boldsymbol{\xi}} \hat{\mathbf{j}}(\boldsymbol{\xi}) \cdot \mathcal{A}(\boldsymbol{\xi}, t) .
$$

where $\hat{H}$ and $\hat{P}_{\mu \nu}$ are the Hamiltonian and the stress tensor operator $^{36,40}$ calculated at Euclidean metric and zero vector potential. The reader is reminded that the effective vector potential $\mathcal{A}(\boldsymbol{\xi}, t)$ in Eq. (26) is determined self-consistently from the zero-current condition of Eq. (17). Hence the only "external" parameter that controls the linear dynamics is the strain tensor $u_{\mu \nu}(\boldsymbol{\xi}, t)$. 
The problem we have formulated in the previous section is amenable to treatment by standard linear response theory. ${ }^{2,41}$ Indeed, we can use the Kubo formalism ${ }^{2}$ to calculate the expectation values in Eqs. (17) and (19). Assuming that the strain tensor $u_{\mu \nu}$ is periodic in time with angular frequency $\omega$, we can write for the stress tensor

$$
\tilde{P}_{\mu \nu}(\boldsymbol{\xi}, \omega)=P_{\mu \nu}^{\mathrm{eq}}(\boldsymbol{\xi})+\int d \boldsymbol{\xi}^{\prime} \tilde{Q}_{\mu \nu \alpha \beta}\left(\boldsymbol{\xi}, \boldsymbol{\xi}^{\prime}, \omega\right) u_{\alpha \beta}\left(\boldsymbol{\xi}^{\prime}, \omega\right),
$$

where $P_{\mu \nu}^{\mathrm{eq}}(\boldsymbol{\xi})$ is the equilibrium stress tensor calculated in the ground state of $\hat{H}$,

$$
P_{\mu \nu}^{\mathrm{eq}}(\boldsymbol{\xi})=\left\langle\Phi(0)\left|\hat{P}_{\mu \nu}(\boldsymbol{\xi})\right| \Phi(0)\right\rangle,
$$

and $\widetilde{Q}_{\mu \nu \alpha \beta}\left(\boldsymbol{\xi}, \boldsymbol{\xi}^{\prime}, \omega\right)$ is the tensor of elasticity in the comoving frame - a fourth-rank tensor with, in general, 21 independent components.

Looking at Eqs. (20)-(23) for the stress tensor operator, we see that there are two qualitatively different contributions to the linear response of $\widetilde{P}_{\mu \nu}$ [i.e., the second term on the right-hand side of Eq. (27)].

The first contribution arises from the fact that the stress tensor operator has an explicit dependence on the displacement field via the metrics $g_{\mu \nu}$. The dependence of $\hat{\widetilde{P}}_{\mu \nu}$ on $g_{\mu \nu}$ is instantaneous in time (i.e., a change in metric is immediately reflected in the stress tensor) and is represented by a frequency-independent contribution to the tensor of elasticity:

$$
\widetilde{P}_{\mu \nu}^{(1)}(\boldsymbol{\xi}, \omega)=\int d \xi^{\prime} \tilde{Q}_{\mu \nu \alpha \beta}^{\infty}\left(\boldsymbol{\xi}, \boldsymbol{\xi}^{\prime}\right) u_{\alpha \beta}\left(\boldsymbol{\xi}^{\prime}, \omega\right)
$$

Formally $\widetilde{Q}_{\mu \nu \alpha \beta}^{\infty}\left(\boldsymbol{\xi}, \boldsymbol{\xi}^{\prime}\right)$ is the expectation value of the functional derivative of $\hat{\widetilde{P}}_{\mu \nu}(\boldsymbol{\xi})$ with respect to $g_{\alpha \beta}\left(\boldsymbol{\xi}^{\prime}\right)$, evaluated at the Euclidean metrics $\left(g_{\mu \nu}=\delta_{\mu \nu}\right)$ :

$$
\tilde{Q}_{\mu \nu \alpha \beta}^{\infty}\left(\boldsymbol{\xi}, \boldsymbol{\xi}^{\prime}\right)=\left\langle\frac{\delta \hat{\tilde{P}}_{\mu \nu}(\boldsymbol{\xi})}{\delta g_{\alpha \beta}\left(\xi^{\prime}\right)}\right\rangle_{g=1} .
$$

We have adopted the superscript " $\infty$ " to emphasize that this part of the tensor of elasticity, being frequency independent, will not go to zero in the limit of infinite frequency. In fact, we will see that this is the only contribution in the high-frequency limit. Note that there is no contribution coming from the explicit dependence of $\widetilde{T}_{\mu \nu}(\xi, t)$ upon the vector potential [not shown in Eq. (22)]. This is because the response of the kinetic stress tensor to the change in vector potential $\mathcal{A}$ (i.e., $\delta \widetilde{T}_{\mu \nu} \propto j_{\mu} \delta \mathcal{A}_{\nu}+j_{\nu} \delta \mathcal{A}_{\mu}$ ) vanishes due to the zero expectation value of the current $\left(\left\langle\hat{j}_{\mu}\right\rangle=0\right)$. Evaluation of the derivative of the stress tensor $\widetilde{P}_{\mu \nu}(\xi, \omega)$ with respect to the deformation tensor $g_{\alpha \beta}$ at the ground state of $\hat{H}_{0}$ and Euclidean metrics leads to

$$
\begin{aligned}
& \widetilde{Q}_{\mu \nu \alpha \beta}^{\infty}=\left\{\left.\frac{\delta_{\alpha \beta}}{2 m} \partial_{\mu} \partial_{\nu^{\prime}} \widetilde{\rho}_{1}^{\mathrm{eq}}\left(\boldsymbol{\xi}, \boldsymbol{\xi}^{\prime}\right)\right|_{\xi^{\prime}=\boldsymbol{\xi}}-\frac{\delta_{\mu \nu}}{4 m} \partial_{\alpha} \partial_{\beta} \widetilde{n}(\boldsymbol{\xi})\right. \\
& +\frac{1}{8 m}\left(\delta_{\mu \alpha} \delta_{\nu \beta}+\delta_{\nu \alpha} \delta_{\mu \beta}-\delta_{\mu \nu} \delta_{\alpha \beta}\right) \nabla^{2} \widetilde{n}(\boldsymbol{\xi}) \\
& -\frac{1}{2}\left(\frac{1}{2} \delta_{\alpha \beta} \delta_{\mu i} \delta_{\nu j}-\delta_{\alpha \mu} \delta_{\beta i} \delta_{\nu j}-\delta_{\alpha \nu} \delta_{\beta j} \delta_{\mu i}\right) \\
& \times \int d \boldsymbol{\xi}^{\prime} \frac{\xi_{i}^{\prime} \xi_{j}^{\prime}}{\left|\boldsymbol{\xi}^{\prime}\right|} \frac{\partial w\left(\left|\boldsymbol{\xi}^{\prime}\right|\right)}{\partial\left|\boldsymbol{\xi}^{\prime}\right|} \widetilde{\rho}_{2}^{\mathrm{eq}}\left(\boldsymbol{\xi}, \boldsymbol{\xi}^{\prime}\right)-\frac{3}{4} \\
& \left.\times \int d \boldsymbol{\xi}^{\prime} \frac{\xi_{\mu}^{\prime} \xi_{\nu}^{\prime} \xi_{\alpha}^{\prime} \xi_{\beta}^{\prime}}{\left|\boldsymbol{\xi}^{\prime}\right|^{3}} \frac{\partial w\left(\left|\xi^{\prime}\right|\right)}{\partial\left|\boldsymbol{\xi}^{\prime}\right|} \widetilde{\rho}_{2}^{\mathrm{eq}}\left(\boldsymbol{\xi}, \boldsymbol{\xi}^{\prime}\right)\right\} \delta\left(\boldsymbol{\xi}-\boldsymbol{\xi}^{\prime}\right) .
\end{aligned}
$$

The second contribution to the linear response of $\widetilde{P}_{\mu \nu}$ comes from the fact that the state in which we calculate the average of $\hat{P}_{\mu \nu}$ is not the ground state but a time-dependent state which evolves under the action of $\hat{\widetilde{H}}(t)$. In calculating this part of the response we just set $g=1$ and $\mathcal{A}=0$ in the stress tensor operator. Thus we have

$$
\widetilde{P}_{\mu \nu}^{(2)}(\boldsymbol{\xi}, \omega)=\int d \boldsymbol{\xi}^{\prime} \Delta \tilde{Q}_{\mu \nu \alpha \beta}\left(\boldsymbol{\xi}, \boldsymbol{\xi}^{\prime}, \omega\right) u_{\alpha \beta}\left(\boldsymbol{\xi}^{\prime}, \omega\right),
$$

where $\Delta \tilde{Q}_{\mu \nu \alpha \beta}\left(\boldsymbol{\xi}, \boldsymbol{\xi}^{\prime}, \omega\right)$ is a combination of the stress-stress response function and the stress-current response functions evaluated with the Hamiltonian $\hat{\tilde{H}}(t)$. Its formal expression is

$$
\begin{aligned}
\Delta \tilde{Q}_{\mu \nu \alpha \beta}\left(\boldsymbol{\xi}, \boldsymbol{\xi}^{\prime}, \omega\right)= & \frac{1}{2}\left\langle\left\langle\hat{P}_{\mu \nu}(\boldsymbol{\xi}) ; \hat{P}_{\alpha \beta}\left(\boldsymbol{\xi}^{\prime}\right)\right\rangle\right\rangle_{\omega}+\int d \xi^{\prime \prime} \\
& \times\left.\left\langle\left\langle\hat{P}_{\mu \nu}(\boldsymbol{\xi}) ; \hat{j}_{\gamma}\left(\xi^{\prime \prime}\right)\right\rangle\right\rangle_{\omega} \frac{\delta \mathcal{A}_{\gamma}\left(\xi^{\prime \prime}\right)}{\delta u_{\alpha \beta}\left(\xi^{\prime}\right)}\right|_{u_{\alpha \beta}=0} .
\end{aligned}
$$

The first term on the right-hand side in Eq. (33) comes from the response to the second term in the Hamiltonian (26). Similarly the second term in Eq. (33) describes the sress produced by the effective vector potential [the response to the third term in the Hamiltonian (26)]. The variational derivative entering the second term is a formal linear response kernel that connects the self-consistent vector potential to the strain tensor:

$$
\mathcal{A}_{\mu}(\boldsymbol{\xi})=\left.\int d \boldsymbol{\xi}^{\prime} \frac{\delta \mathcal{A}_{\mu}(\boldsymbol{\xi})}{\delta u_{\alpha \beta}\left(\xi^{\prime}\right)}\right|_{u_{\alpha \beta}=0} u_{\alpha \beta}\left(\boldsymbol{\xi}^{\prime}\right) .
$$

This variational derivative can be straightforwardly calculated from the linear response version of the self-consistency equation (17):

$$
\begin{aligned}
\mathcal{A}_{\mu}(\boldsymbol{\xi})= & -\frac{i}{n_{0}} \int d \boldsymbol{\xi}^{\prime}\left\langle\left\langle\hat{j}_{\mu}(\boldsymbol{\xi}) ; \hat{j}_{\nu}\left(\boldsymbol{\xi}^{\prime}\right)\right\rangle\right\rangle_{\omega} \mathcal{A}_{\nu}\left(\boldsymbol{\xi}^{\prime}\right) \\
& -\frac{i}{2 n_{0}} \int d \boldsymbol{\xi}^{\prime}\left\langle\left\langle\hat{j}_{\mu}(\boldsymbol{\xi}) ; \hat{P}_{\alpha \beta}\left(\boldsymbol{\xi}^{\prime}\right)\right\rangle\right\rangle_{\omega} u_{\alpha \beta}\left(\boldsymbol{\xi}^{\prime}\right) .
\end{aligned}
$$


The complete formula for the tensor of elasticity in the comoving frame can then be written as

$$
\widetilde{Q}_{\mu \nu \alpha \beta}\left(\boldsymbol{\xi}, \boldsymbol{\xi}^{\prime}, \omega\right)=\widetilde{Q}_{\mu \nu \alpha \beta}^{\infty}\left(\boldsymbol{\xi}, \boldsymbol{\xi}^{\prime}\right)+\Delta \widetilde{Q}_{\mu \nu \alpha \beta}\left(\xi, \xi^{\prime}, \omega\right) .
$$

From the general properties of Kubo response functions we see that $\Delta \widetilde{Q}_{\mu \nu \alpha \beta}\left(\boldsymbol{\xi}, \boldsymbol{\xi}^{\prime}, \omega\right)$ vanishes in the high-frequency limit (so does the self-consistent vector potential $\mathcal{A}$ ). This confirms that $\widetilde{Q}_{\mu \nu \alpha \beta}^{\infty}\left(\boldsymbol{\xi}, \boldsymbol{\xi}^{\prime}\right)$ is indeed the correct highfrequency limit of the tensor of elasticity.

Finally, we must transform the stress tensor back to the laboratory frame, according to the transformation rule of Eq. (25). Since $\widetilde{P}_{\mu \nu}^{(1)}(\xi, \omega)$ and $\widetilde{P}_{\mu \nu}^{(2)}(\xi, \omega)$ are already linear in the displacement field, they are not altered by the coordinate thansformation: all we need to do is replace $\boldsymbol{\xi}$ by $\mathbf{r}$. However, the equilibrium term, $P_{\mu \nu}^{\mathrm{eq}}(\boldsymbol{\xi})$ of Eq. (27), does generate linear-order contributions when substituted in the transformation of Eq. (25). Making use of Eqs. (27) and (10), we obtain two additional contributions

$$
P_{\mu \nu}^{(3)}(\mathbf{r}, \omega)=-\left(\delta_{\alpha \mu} \frac{\partial u_{\beta}}{\partial r_{\nu}}+\delta_{\beta \nu} \frac{\partial u_{\alpha}}{\partial r_{\mu}}\right) P_{\alpha \beta}^{\mathrm{eq}}(\mathbf{r})
$$

and

$$
P_{\mu \nu}^{(4)}(\mathbf{r}, \omega)=-u_{\alpha} \frac{\partial}{\partial r_{\alpha}} P_{\mu \nu}^{\mathrm{eq}}(\mathbf{r})
$$

The last contribution comes from the fact that in transforming back to the laboratory frame, we first replace the argument $\boldsymbol{\xi}$ by $\mathbf{r}-\mathbf{u}$ and then make a Taylor expansion of $\widetilde{P}_{\mu \nu}(\boldsymbol{\xi}, \omega)$ about $\mathbf{u}=0$. This contribution can also be expressed as

$$
P_{\mu \nu}^{(4)}(\mathbf{r}, \omega)=-\int d^{3} r^{\prime} \mathbf{u} \cdot \nabla_{\mathbf{r}^{\prime}} n_{0}\left(\mathbf{r}^{\prime}\right) \frac{\delta P_{\mu \nu}^{\mathrm{eq}}\left(\left[n_{0}\right] ; \mathbf{r}\right)}{\delta n_{0}\left(\mathbf{r}^{\prime}\right)},
$$

where we have used the fact that the equilibrium stress tensor $P_{\mu \nu}^{\mathrm{eq}}$ is a translationally invariant functional of the groundstate density.

Finally, the linear response of the stress tensor is the sum of the four contributions given by Eqs. (29), (32), (36), and (37).

\section{EXCHANGE-CORRELATION STRESS TENSOR}

Since the noninteracting kinetic part and the Hartree potential part are usually treated exactly in the time-dependent Kohn-Sham equation, we only focus in what follows on approximating the $\mathrm{XC}$ stress tensor, which has two contributions: the kinetic part and the interaction part. The XC part of the kinetic stress tensor is defined as the difference between interacting and noninteracting kinetic stress tensors. (Notice that, unlike the interaction part of the kinetic energy, which has no exchange contribution, the interaction part of the kinetic stress tensor has both exchange and correlation contributions.) Precisely, in the comoving frame we have

$$
\tilde{T}_{\mathrm{xc}, \mu \nu}(\boldsymbol{\xi}, \omega)=\widetilde{T}_{\mu \nu}(\boldsymbol{\xi}, \omega)-\widetilde{T}_{\mu \nu}^{\mathrm{S}}(\boldsymbol{\xi}, \omega),
$$

where $\widetilde{T}_{\mu \nu}^{\mathrm{S}}$ is the kinetic stress tensor of a noninteracting system with the density $n_{0}(\xi)$ of the interacting system.
The XC part of the interaction stress tensor, denoted by $\widetilde{W}_{\mathrm{xc}, \mu \nu}$, is obtained from the interaction stress tensor of Eq. (23) after subtracting the Hartree contribution-i.e., after replacing the two-particle density matrix $\widetilde{\rho}_{2}\left(\boldsymbol{\xi}, \boldsymbol{\xi}^{\prime}, t\right)$ by

$$
\tilde{n}_{0}(\xi) \tilde{\rho}_{\mathrm{xc}}\left(\xi, \boldsymbol{\xi}^{\prime}, t\right) \equiv \tilde{\rho}_{2}(\boldsymbol{\xi}, \boldsymbol{\xi}, t)-\tilde{n}_{0}(\boldsymbol{\xi}) \tilde{n}_{0}(\boldsymbol{\xi}) .
$$

The full XC stress tensor is then given by

$$
\widetilde{P}_{\mu \nu}^{\mathrm{xc}}(\boldsymbol{\xi}, \omega)=\widetilde{T}_{\mathrm{xc}, \mu \nu}(\boldsymbol{\xi}, \omega)+\widetilde{W}_{\mathrm{xc}, \mu \nu}(\boldsymbol{\xi}, \omega)
$$

In linear response theory, it is given by an equation completely analogous to Eq. (27):

$$
\widetilde{P}_{\mu \nu}^{\mathrm{xc}}(\boldsymbol{\xi}, \omega)=P_{\mu \nu}^{\mathrm{xc}, \mathrm{eq}}(\boldsymbol{\xi})+\int d \xi^{\prime} \widetilde{Q}_{\mu \nu \alpha \beta}^{\mathrm{xc}}\left(\boldsymbol{\xi}, \boldsymbol{\xi}^{\prime}, \omega\right) u_{\alpha \beta}\left(\boldsymbol{\xi}^{\prime}, \omega\right)
$$

where $P_{\mu \nu}^{\mathrm{xc}, \mathrm{eq}}(\boldsymbol{\xi})$ is the XC stress tensor [Eq. (41)] evaluated at Euclidean metric and zero vector potential (i.e., at the ground state) and $\widetilde{Q}_{\mu \nu \alpha \beta}^{\mathrm{xc}}\left(\boldsymbol{\xi}, \boldsymbol{\xi}^{\prime}, \omega\right)=\widetilde{Q}_{\mu \nu \alpha \beta}^{\mathrm{xc}, \infty}\left(\boldsymbol{\xi}, \boldsymbol{\xi}^{\prime}\right)+\Delta \widetilde{Q}_{\mu \nu \alpha \beta}^{\mathrm{xc}}\left(\boldsymbol{\xi}, \boldsymbol{\xi}^{\prime}, \omega\right)$. Here $\widetilde{Q}_{\mu \nu \alpha \beta}^{\mathrm{xc}, \infty}$ is the infinite-frequency part of the tensor of elasticity evaluated at the ground state. $\Delta \widetilde{Q}_{\mu \nu \alpha \beta}^{\mathrm{xc}}$ is the finitefrequency part. The terms arising from the transformation to the laboratory frame are still given by Eqs. (36)-(38), with the full stress tensor $P_{\mu \nu}^{\mathrm{eq}}$ replaced by the XC part $P_{\mu \nu}^{\mathrm{xc}, \mathrm{eq}}$.

\section{HIGH-FREQUENCY LIMIT}

Obviously, the tensor of elasticity is a very complicated object. Even after taking into account the symmetries $Q_{\mu \nu \alpha \beta}=Q_{\nu \mu \alpha \beta}=Q_{\mu \nu \beta \alpha}=Q_{\alpha \beta \mu \nu}$, there are still in general 21 independent components. ${ }^{42}$ Each component is a function of $\boldsymbol{\xi}$ and $\boldsymbol{\xi}^{\prime}$, as well as frequency. It is clear that, in order to make progress, some rather drastic approximation is needed.

In this section we begin to develop an approximation scheme on the premise that the $\mathrm{XC}$ part of the one-particle density matrix and the XC hole can be locally replaced by their spherical average. A similar approximation ${ }^{43-45}$ is implicit in many state-of-the-art methods for calculating the electronic structure, such as the GGA and the meta-GGA. To begin with, we completely disregard the range of nonlocality of $\widetilde{Q}_{\mu \nu \alpha \beta}^{\mathrm{xc}}\left(\boldsymbol{\xi}, \boldsymbol{\xi}^{\prime}\right)$-i.e., the fact that it depends on two spatial arguments. The possibility of this approximation depends only on the assumed slow variation of the strain tensor. Then, by making a local spherical approximation, we will reduce the number of independent components of $\widetilde{Q}_{\mu \nu \alpha \beta}^{\mathrm{xc}}$ from 21 to 2 -namely, the local bulk modulus and the local shear modulus. The only difference from the conventional LDA will be that the local viscoelastic constants depend not only on the local density, but also on its derivatives and other inhomogeneity parameters. In the high-frequency limit, the whole scheme is justified a posteriori from the fact that the calculation of $\widetilde{Q}^{\mathrm{xc}, \infty}$ is really a static ground-state calculation, for which commonly used semilocal functionals of the static DFT should be quite accurate. In the following, we will examine the construction in greater detail. 


\section{A. Three approximations}

(i) In the static DFT, the XC hole underlying a semilocal functional is usually constructed to simulate the spherical average $^{44-46}$ of the exact hole $\rho_{\mathrm{xc}}(\mathbf{r}, \mathbf{r}+\mathbf{u})$,

$$
\rho_{\mathrm{xc}}(\mathbf{r}, u) \equiv \int \frac{d \Omega_{\mathbf{u}}}{4 \pi} \rho_{\mathrm{xc}}(\mathbf{r}, \mathbf{r}+\mathbf{u}) .
$$

The justification for this is that the XC energy does not depend upon the details of the angular dependence of the hole. To make a connection with the static DFT, we assume here that we can locally replace the exact XC hole $\widetilde{\rho}_{\mathrm{xc}}\left(\boldsymbol{\xi}, \boldsymbol{\xi}^{\prime}\right)$ in the comoving frame, defined in Eq. (40), with its spherical average over the separation vector $\xi^{\prime}$ :

$$
\tilde{\rho}_{\mathrm{xc}}\left(\boldsymbol{\xi}, \xi^{\prime}\right) \equiv \int \frac{d \Omega_{\xi^{\prime}}}{4 \pi} \tilde{\rho}_{\mathrm{xc}}\left(\boldsymbol{\xi}, \boldsymbol{\xi}^{\prime}\right) .
$$

With this local spherical-symmetry assumption, the interaction part of the equilibrium XC stress tensor is immediately simplified to

$$
W_{\mathrm{xc}, \mu \nu}^{\mathrm{eq}}(\boldsymbol{\xi})=\frac{1}{3} \delta_{\mu \nu} W_{\mathrm{xc}}(\boldsymbol{\xi})
$$

where $W_{\mathrm{xc}}(\boldsymbol{\xi})$ is the expectation value of the potential energy density operator of Eq. (15) calculated at Euclidean metric and zero vector potential minus the Hartree energy.

The spherical-symmetry assumption is exactly true for a slowly varying density, while it is not well satisfied and sometimes even severely violated for a strongly inhomogeneous system. From the performances of a ladder ${ }^{47}$ of density functionals in the static DFT, we see that a density functional may be very successful in practice even though it is not well formally justified for some conditions. ${ }^{48}$ However, this extrapolated assumption leaves us some space for further improvement.

(ii) We further assume that the XC part of the one-particle density matrix, $\tilde{\rho}_{1, \mathrm{xc}}\left(\boldsymbol{\xi}, \boldsymbol{\xi}^{\prime}\right)$, is of spherical symmetry. Under this assumption, we can locally replace $\widetilde{\rho}_{1, \mathrm{xc}}\left(\boldsymbol{\xi}, \boldsymbol{\xi}^{\prime}\right)$ by its spherical average

$$
\tilde{\rho}_{1, \mathrm{xc}}\left(\xi, \xi^{\prime}\right) \equiv \int \frac{d \Omega_{\xi^{\prime}}}{4 \pi} \widetilde{\rho}_{1, \mathrm{xc}}\left(\boldsymbol{\xi}, \boldsymbol{\xi}^{\prime}\right),
$$

where $\widetilde{\rho}_{1, \mathrm{xc}}\left(\boldsymbol{\xi}, \boldsymbol{\xi}^{\prime}\right)$ is defined as the difference between the interacting and noninteracting one-particle density matrices-i.e., $\tilde{\rho}_{1, \mathrm{xc}}\left(\boldsymbol{\xi}, \boldsymbol{\xi}^{\prime}\right)=\tilde{\boldsymbol{\rho}}_{1}\left(\boldsymbol{\xi}, \boldsymbol{\xi}^{\prime}\right)-\widetilde{\boldsymbol{\rho}}_{1}^{\mathrm{S}}\left(\boldsymbol{\xi}, \boldsymbol{\xi}^{\prime}\right)$. Note that $\tilde{\rho}_{1}(\boldsymbol{\xi}, \boldsymbol{\xi})=\widetilde{\rho}_{1}^{\mathrm{S}}(\boldsymbol{\xi}, \boldsymbol{\xi})=\widetilde{n}_{0}(\boldsymbol{\xi})$. Thus the kinetic part of the equilibrium $\mathrm{XC}$ stress tensor can be written as

$$
T_{\mathrm{xc}, \mu \nu}^{\mathrm{eq}}(\xi)=\frac{2}{3} \delta_{\mu \nu} T_{\mathrm{xc}}(\xi) .
$$

$T_{\mathrm{xc}}$ is the XC part of the kinetic energy density functional. We see from the coupling-constant integration formula ${ }^{49}$ that only the correlation part of the kinetic XC stress tensor survives in this approximation. Furthermore, the infinitefrequency part of the complicated tensor of elasticity simplifies to

$$
\begin{aligned}
& \widetilde{Q}_{\alpha \alpha \beta \beta}^{\mathrm{xc} \infty}=\left[3 T_{\mathrm{c}}\left(\left[n_{0}\right] ; \boldsymbol{\xi}\right)+W_{\mathrm{xc}}\left(\left[n_{0}\right] ; \boldsymbol{\xi}\right)\right] \delta\left(\boldsymbol{\xi}-\boldsymbol{\xi}^{\prime}\right), \\
& \widetilde{Q}_{\alpha \beta \alpha \beta}^{\mathrm{xc} \infty}=\left[T_{\mathrm{c}}\left(\left[n_{0}\right] ; \boldsymbol{\xi}\right)-2 W_{\mathrm{xc}}\left(\left[n_{0}\right] ; \boldsymbol{\xi}\right)\right] \delta\left(\boldsymbol{\xi}-\boldsymbol{\xi}^{\prime}\right) .
\end{aligned}
$$

Combining the local spherical approximations for the kinetic and potential parts of exchange-correlation we finally arrive at

$$
P_{\mu \nu}^{\mathrm{eq}}(\boldsymbol{\xi})=\delta_{\mu \nu} P^{\mathrm{xc}, \mathrm{eq}}\left(\left[\tilde{n}_{0}\right], \boldsymbol{\xi}\right),
$$

where $P^{\mathrm{xc}, \mathrm{eq}}$ is the local equilibrium $\mathrm{XC}$ pressure defined as

$$
P^{\mathrm{xc}, \mathrm{eq}}\left(\left[\tilde{n}_{0}\right], \boldsymbol{\xi}\right)=\frac{2 T_{\mathrm{c}}\left(\left[\tilde{n}_{0}\right] ; \boldsymbol{\xi}\right)+W_{\mathrm{xc}}\left(\left[\tilde{n}_{0}\right] ; \boldsymbol{\xi}\right)}{3} .
$$

This expression is then inserted in Eqs. (36) and (38), allowing us to calculate the contributions "(3)" and "(4)" of the $\mathrm{XC}$ stress tensor in the laboratory frame:

$$
\begin{gathered}
P_{\mu \nu}^{\mathrm{xc},(3)}(\mathbf{r}, \omega)=\int d \mathbf{r}^{\prime} Q_{\mu \nu \alpha \beta}^{\mathrm{xc}, \mathrm{t}}\left(\mathbf{r}, \mathbf{r}^{\prime}\right) u_{\alpha \beta}\left(\mathbf{r}^{\prime}, \omega\right), \\
P_{\mu \nu}^{\mathrm{xc},(4)}(\mathbf{r}, \omega)=-\delta_{\mu \nu} \int d^{3} r^{\prime} \mathbf{u} \cdot \nabla_{\mathbf{r}^{\prime}} n_{0}\left(\mathbf{r}^{\prime}\right) \frac{\delta P^{\mathrm{xc}, \mathrm{eq}}(\mathbf{r})}{\delta n_{0}\left(\mathbf{r}^{\prime}\right)},
\end{gathered}
$$

where

$$
Q_{\mu \nu \alpha \beta}^{\mathrm{xc}, \mathrm{t}}=\frac{1}{2} P^{\mathrm{xc}, \mathrm{eq}}(\mathbf{r})\left(\delta_{\mu \alpha} \delta_{\nu \beta}+\delta_{\nu \alpha} \delta_{\mu \beta}\right) \delta\left(\mathbf{r}-\mathbf{r}^{\prime}\right)
$$

is a component of the infinite-frequency part of the tensor of elasticity and $P^{\mathrm{xc}, \mathrm{eq}}$ is defined by Eq. (50). As we shall see in Sec. V B, $P_{\mu \nu}^{\mathrm{xc},(4)}(\mathbf{r}, \omega)$ only contributes to the low-frequency limit of the tensor of elasticity $\Delta Q_{\mu \nu \alpha \beta}^{\mathrm{xc}}$, canceling the lowfrequency limit of the longitudinal part of $\Delta \widetilde{Q}_{\mu \nu \alpha \beta}^{\mathrm{xc}}[$ see Eq. (60)], while $P_{\mu \nu}^{\mathrm{xc},(3)}(\mathbf{r}, \omega)$ contributes to the infinite-frequency part.

The complete infinite-frequency part of the tensor of elasticity in the laboratory frame is obtained as

$$
Q_{\mu \nu \alpha \beta}^{\mathrm{xc}, \infty}=\widetilde{Q}_{\mu \nu \alpha \beta}^{\mathrm{xc}, \infty}\left(\mathbf{r}, \mathbf{r}^{\prime}\right)+Q_{\mu \nu \alpha \beta}^{\mathrm{xc}, \mathrm{t}}\left(\mathbf{r}, \mathbf{r}^{\prime}\right) .
$$

(iii) Finally we assume that the strain tensor of Eq. (4) is slowly varying in space so that its spatial derivative can be neglected. Notice that we are not assuming that the groundstate density $n_{0}(\mathbf{r})$ is slowly varying, only that the strain tensor (the deformation) is. With this assumption, together with the two local spherical-symmetry assumptions described above, we are able to express the infinite-frequency part of the tensor of elasticity in terms of only two quantities $K_{\mathrm{xc}}^{\infty}$ and $\mu_{\mathrm{xc}}^{\infty}$ :

$$
\begin{aligned}
Q_{\mu \nu \alpha \beta}^{\mathrm{xc}, \infty}\left(\mathbf{r}, \mathbf{r}^{\prime}\right)= & {\left[\frac{K_{\mathrm{xc}}^{\infty}(\mathbf{r})}{2} \delta_{\mu \nu} \delta_{\alpha \beta}+\mu_{\mathrm{xc}}^{\infty}(\mathbf{r})\left(-\frac{\delta_{\mu \nu} \delta_{\alpha \beta}}{3}\right.\right.} \\
& \left.\left.+\frac{\delta_{\mu \alpha} \delta_{\nu \beta}+\delta_{\nu \alpha} \delta_{\mu \beta}}{2}\right)\right] \delta\left(\mathbf{r}-\mathbf{r}^{\prime}\right),
\end{aligned}
$$

where $K_{\mathrm{xc}}^{\infty}$ and $\mu_{\mathrm{xc}}^{\infty}$ are the local bulk and shear moduli of the inhomogeneous system given by Eq. (7). To arrive at Eq. (55), we just substitute Eqs. (48) and (53) into Eq. (54). 


\section{B. Subtraction of the adiabatic contribution}

Let us summarize the situation at this point. The linear response of the XC stress tensor is the sum of four contributions:

$$
\begin{aligned}
P_{\mu \nu}^{\mathrm{xc}}(\mathbf{r}, \omega)= & \delta_{\mu \nu} P^{\mathrm{xc}, \mathrm{eq}}\left(\left[n_{0}\right] ; \mathbf{r}\right) \\
& -\delta_{\mu \nu} \int d^{3} r^{\prime} \mathbf{u} \cdot \nabla_{\mathbf{r}^{\prime}} n_{0}\left(\mathbf{r}^{\prime}\right) \frac{\delta P^{\mathrm{xc}, \mathrm{eq}}\left(\left[n_{0}\right] ; \mathbf{r}\right)}{\delta n_{0}\left(\mathbf{r}^{\prime}\right)} \\
& +\left[Q_{\mu \nu \alpha \beta}^{\mathrm{xc}, \infty}(\mathbf{r})+\Delta \widetilde{Q}_{\mu \nu \alpha \beta}^{\mathrm{xc}}(\mathbf{r}, \omega)\right] u_{\alpha \beta}(\mathbf{r}, \omega),
\end{aligned}
$$

where $Q_{\mu \nu \alpha \beta}^{\mathrm{xc}, \infty}(\mathbf{r})$ is the quantity in the square brackets of Eq. (55).

Define the adiabatic approximation for the XC stress tensor as

$$
P_{\mu \nu}^{\mathrm{xc}, \mathrm{ad}}(\mathbf{r}, t)=P_{\mu \nu}^{\mathrm{xc}, \mathrm{eq}}([n] ; \mathbf{r}, t),
$$

where $n \equiv n(\mathbf{r}, t)$ is the instantaneous density and $P_{\mu \nu}^{\mathrm{xc}, \mathrm{eq}}([n] ; \mathbf{r}, t)$ is the equilibrium XC stress tensor with $n_{0}(\mathbf{r})$ replaced by $n(\mathbf{r}, t)$. In linear response,

$$
n(\mathbf{r}, t)=n_{0}(\mathbf{r})-\nabla \cdot\left[n_{0}(\mathbf{r}) \mathbf{u}(\mathbf{r}, t)\right] .
$$

Linearization of the adiabatic approximation, together with the local-symmetry assumptions of Eqs. (44) and (46), yields

$$
\begin{aligned}
P_{\mu \nu}^{\mathrm{xc}, \mathrm{ad}}(\mathbf{r}, \omega)= & \delta_{\mu \nu} P^{\mathrm{xc}, \mathrm{eq}}\left(\left[n_{0}\right] ; \mathbf{r}\right) \\
& -\delta_{\mu \nu} \int d^{3} r^{\prime} \nabla^{\prime} \cdot\left[n_{0}\left(\mathbf{r}^{\prime}\right) \mathbf{u}\left(\mathbf{r}^{\prime}, \omega\right)\right] \frac{\delta P^{\mathrm{xc}, \mathrm{eq}}\left(\left[n_{0}\right] ; \mathbf{r}\right)}{\delta n_{0}\left(\mathbf{r}^{\prime}\right)} .
\end{aligned}
$$

Finally, subtracting Eq. (59) from Eq. (56) we obtain the linear-order correction to the adiabatic stress tensor in the laboratory frame:

$$
\begin{aligned}
\Delta P_{\mu \nu}^{\mathrm{xc}}(\mathbf{r}, \omega)= & {\left[Q_{\mu \nu \alpha \beta}^{\mathrm{xc}, \infty}(\mathbf{r})+\Delta \widetilde{Q}_{\mu \nu \alpha \beta}^{\mathrm{xc}}(\mathbf{r}, \omega)\right] u_{\alpha \beta}(\mathbf{r}, \omega) } \\
& -\frac{\delta_{\mu \nu}}{2} K_{\mathrm{xc}}^{0}(\mathbf{r}) u_{\alpha \alpha} .
\end{aligned}
$$

Here we have again made use of the assumption of slow variation of the strain to pull $\mathbf{u}(\mathbf{r}, \omega)$ out of the integral (notice that $\left.u_{\alpha \alpha}=-2 \nabla \cdot \mathbf{u}\right) . K_{\mathrm{xc}}^{0}(\mathbf{r})$ is the zero-frequency limit of the $\mathrm{XC}$ bulk modulus defined by

$$
K_{\mathrm{xc}}^{0}(\mathbf{r}) \equiv \int d^{3} r^{\prime} n_{0}\left(\mathbf{r}^{\prime}\right) \frac{\delta P^{\mathrm{xc}, \mathrm{eq}}\left(\left[n_{0}\right] ; \mathbf{r}\right)}{\delta n_{0}\left(\mathbf{r}^{\prime}\right)}
$$

and can be calculated from the static DFT, as discussed in the next subsection. In the uniform-electron-gas limit, $P^{\mathrm{xc}, \mathrm{eq}}$ is simply a function of the ground-state density and $K_{\mathrm{xc}}^{0}(\mathbf{r})$ reduces to $K_{\mathrm{xc}}^{\mathrm{h}, 0}\left(n_{0}\right)$ of Eq. (5).

The tilde used for the $r$-dependent tensor of elasticity in Eqs. (54), (56), and (60) is only a reminder that these quantities are calculated in the comoving frame. In linear response, they can be considered as a function of either $\boldsymbol{\xi}$ or $\mathbf{r}$.

Imposing the two local spherical-symmetry assumptions and the local deformation approximation on the finitefrequency part of the tensor of elasticity, we finally have

$$
\begin{aligned}
\Delta Q_{\mu \nu \alpha \beta}^{\mathrm{xc}}(\mathbf{r}, \omega)= & \frac{\Delta K_{\mathrm{xc}}(\mathbf{r}, \omega)}{2} \delta_{\mu \nu} \delta_{\alpha \beta}+\Delta \mu_{\mathrm{xc}}(\mathbf{r}, \omega) \\
& \times\left(-\frac{\delta_{\mu \nu} \delta_{\alpha \beta}}{3}+\frac{\delta_{\mu \alpha} \delta_{\nu \beta}+\delta_{\nu \alpha} \delta_{\mu \beta}}{2}\right) .
\end{aligned}
$$

The finite-frequency parts of the bulk and shear moduli $\Delta K_{\mathrm{xc}}(\mathbf{r}, \omega)$ and $\Delta \mu_{\mathrm{xc}}(\mathbf{r}, \omega)$ will be constructed in the next section.

\section{Construction of $K_{x c}^{\infty}, \mu_{x c}^{\infty}$, and $K_{x c}^{0}$ from the static DFT}

The frequency-independent parts of the bulk and shear moduli $K_{x c}^{\infty}, \mu_{x c}^{\infty}$, and $K_{x c}^{0}$ given by Eqs. (7) and (61), respectively, are expressed in terms of the kinetic correlation and XC potential energy density densities $T_{\mathrm{c}}(\mathbf{r})$ and $W_{\mathrm{xc}}(\mathbf{r})$ of the ground state. 49

In the static DFT, $T_{\mathrm{c}}(\mathbf{r})$ and $W_{\mathrm{xc}}(\mathbf{r})$ can be constructed from the coupling-constant average $n_{0} \epsilon_{\mathrm{xc}}\left(\left[n_{0}\right] ; \mathbf{r}\right)$ via a coupling-constant integration formula. ${ }^{50,51}$ For example, within the LDA, GGA, ${ }^{52}$ or meta-GGA functional, ${ }^{53}$ we have

$$
\begin{gathered}
T_{\mathrm{c}}\left(\left[n_{0}\right] ; \mathbf{r}\right)=-n_{0} \frac{\partial}{\partial r_{s}}\left[r_{s} \epsilon_{\mathrm{c}}\left(r_{s}, s_{1}, s_{2}, \ldots\right)\right], \\
W_{\mathrm{xc}}(\mathbf{r})=n_{0} \epsilon_{\mathrm{xc}}+n_{0} \frac{\partial}{\partial r_{s}}\left[r_{s} \epsilon_{\mathrm{c}}\left(r_{s}, s_{1}, s_{2}, \ldots\right)\right],
\end{gathered}
$$

where $r_{s}(\mathbf{r})=a_{0}^{-1}\left(3 / 4 \pi n_{0}\right)^{1 / 3}, a_{0}$ is the Bohr radius, and $s_{1}, s_{2}, \ldots$ are dimensionless variables,

$$
\begin{gathered}
s_{1}=s=\left|\nabla n_{0}\right| / 2 k_{\mathrm{F}} n_{0}, \\
s_{2}=T_{W}(\mathbf{r}) / T(\mathbf{r}) .
\end{gathered}
$$

Here $T_{W}=\left|\nabla n_{0}\right|^{2} / 8 n_{0}$ is the von Weizsäcker kinetic energy density and $T(\mathbf{r})$ is the Kohn-Sham orbital kinetic energy density. Therefore, the knowledge of $\epsilon_{\mathrm{xc}}(\mathbf{r})$ enables us to estimate $T_{\mathrm{c}}(\mathbf{r})$ and $W_{\mathrm{xc}}(\mathbf{r})$. In particular, the meta-GGA functional has achieved consistent accuracy in both condensed matter physics ${ }^{48}$ and quantum chemistry ${ }^{54,55}$ and has been extended to describe systems in a magnetic field ${ }^{56,57}$ or systems formed with van der Waals interactions. ${ }^{58}$ This completes our construction of the infinite-frequency part of the $\mathrm{XC}$ elastic moduli of Eq. (7).

In addition $K_{\mathrm{xc}}^{0}$ can be obtained by substituting Eqs. (50), (63), and (64) into Eq. (61). As an illustrative example, we calculate it within the GGA. The result is shown in the Appendix.

\section{FREQUENCY DEPENDENCE}

The most challenging task in this work is the calculation of the truly dynamical part of the tensor of elasticity $\Delta \widetilde{Q}_{\mu \nu \alpha \beta}^{x c}\left(\boldsymbol{\xi}, \boldsymbol{\xi}^{\prime}, \omega\right)$ of Eq. (32). This has been expressed in Eq. (62) in terms of two dynamical viscoelastic coefficients $\Delta K_{x c}(\omega)$ and $\Delta \mu_{x c}(\omega)$ (to lighten the notation, the dependence on $\mathbf{r}$ via the local density and its derivatives has been suppressed). A full-fledged calculation of these coefficients is out of our reach for the time being. However, we propose an 
approximate expression for the frequency dependence of $\Delta K_{x c}(\omega)$ and $\Delta \mu_{x c}(\omega)$, based on the following facts.

First we notice that (like all causal response functions) $\Delta K_{x c}(\omega)$ and $\Delta \mu_{x c}(\omega)$ satisfy the dispersion relations

$$
\begin{aligned}
& \operatorname{Re} K_{\mathrm{xc}}(\omega)-K_{\mathrm{xc}}^{\infty}=\mathrm{P} \int_{-\infty}^{\infty} \frac{d \omega^{\prime}}{\pi} \frac{\operatorname{Im} \Delta K_{\mathrm{xc}}\left(\omega^{\prime}\right)}{\omega^{\prime}-\omega}, \\
& \operatorname{Re} \mu_{\mathrm{xc}}(\omega)-\mu_{\mathrm{xc}}^{\infty}=\mathrm{P} \int_{-\infty}^{\infty} \frac{d \omega^{\prime}}{\pi} \frac{\operatorname{Im} \Delta \mu_{\mathrm{xc}}\left(\omega^{\prime}\right)}{\omega^{\prime}-\omega},
\end{aligned}
$$

where $\mathrm{P}$ represents the Cauchy principal value. So the problem is "reduced" to the calculation of the imaginary parts of $\Delta K_{x c}(\omega)$ and $\Delta \mu_{x c}(\omega)$. Since $\operatorname{Im} \Delta K_{x c}(\omega)=\operatorname{Im} K_{x c}(\omega)$ and $\operatorname{Im} \Delta \mu_{x c}(\omega)=\operatorname{Im} \mu_{x c}(\omega)$, the real parts of $K_{\text {xc }}$ and $\mu_{\text {xc }}$ in Eqs. (67) and (68) include both infinite-frequency $\left(K_{\mathrm{xc}}^{\infty}\right.$ and $\left.\mu_{\mathrm{xc}}^{\infty}\right)$ and finite-frequency $\left[\operatorname{Re} \Delta K_{\mathrm{xc}}(\omega)\right.$ and $\left.\operatorname{Re} \Delta \mu_{\mathrm{xc}}(\omega)\right]$ contributions. Furthermore, it follows from Eqs. (67) and (68) that these imaginary parts satisfy the sum rules

$$
\begin{aligned}
& K_{\mathrm{xc}}^{0}-K_{\mathrm{xc}}^{\infty}=\int_{-\infty}^{\infty} \frac{d \omega}{\pi} \frac{\operatorname{Im} \Delta K_{\mathrm{xc}}(\omega)}{\omega}, \\
& \mu_{\mathrm{xc}}^{0}-\mu_{\mathrm{xc}}^{\infty}=\int_{-\infty}^{\infty} \frac{d \omega}{\pi} \frac{\operatorname{Im} \Delta \mu_{\mathrm{xc}}(\omega)}{\omega},
\end{aligned}
$$

where $K_{\mathrm{xc}}^{\infty}$ and $\mu_{\mathrm{xc}}^{\infty}$ have been constructed in Secs. V B and $\mathrm{V} \mathrm{C}$. The idea then is to choose reasonable forms for the frequency dependences of $\operatorname{Im} \Delta K_{x c}(\omega)$ and $\operatorname{Im} \Delta \mu_{x c}(\omega)$, such that they satisfy Eqs. (69) and (70), respectively, and correctly reduce to the uniform-gas limits in a system of slowly varying density.

The choice of these forms is heavily influenced by wellknown results of the theory of the homogeneous electron liquid. For a system of uniform density in the limit of zero frequency, $\operatorname{Im} \Delta \mu_{x c}(\omega) \sim \omega$ and $\operatorname{Im} \Delta K_{x c}(\omega) \sim \omega^{3}$ for $\omega \rightarrow 0$. For large frequency, on the other hand, both $\operatorname{Im} \Delta \mu_{x c}(\omega)$ and $\operatorname{Im} \Delta K_{x c}(\omega)$ decrease as $\omega^{-3 / 2}$. We assume that these behaviors remain valid in the inhomogeneous system, with coefficients that depend only on the local density. Thus we neglect gradient corrections to both the high- and low-frequency parts of the viscosity spectra. This, however, does not mean that $\operatorname{Im} \Delta K_{x c}(\omega)$ and $\operatorname{Im} \Delta \mu_{x c}(\omega)$ are free of gradient corrections. In the homogeneous electron gas both spectra exhibit considerable structure at intermediate frequency (about twice the plasmon frequency $\omega_{p}$ ), and the formulas introduced in Ref. 61 take this into account through a term designed to produce a peak at $2 \omega_{p}$. A similar term must also be introduced in the present interpolation formulas, if we wish them to reduce to the homogenous electron gas formulas in the limit $s \rightarrow 0$. But the sum rules Eqs. (69) and (70) force the coefficient of this term to be a function of $s$ : this is the mechanism through which our inhomogeneous spectra become $s$ dependent at intermediate frequency.

More precisely, our interpolation formulas for $\operatorname{Im} \Delta K_{x c}(\omega)$ and $\operatorname{Im} \Delta \mu_{x c}(\omega)$ are based on the following four conditions: (i) in the high-frequency limit, second-order perturbation theory ${ }^{59,60}$ gives $\operatorname{Im} \Delta K_{\mathrm{xc}}=-n_{0}^{2} \pi m e^{4} / 9(m \omega)^{3 / 2}$ and $\operatorname{Im} \Delta \mu_{\mathrm{xc}}$
$=-16 n_{0}^{2} \pi m e^{4} / 15(m \omega)^{3 / 2}$; (ii) in the low-frequency limit, $\operatorname{Im} \Delta K_{\mathrm{xc}} \quad$ vanishes as $O\left(\omega^{3}\right)$, while $\operatorname{Im} \Delta \mu_{\mathrm{xc}}=$ $-\omega\left(m e^{2} / \pi\right)^{2} k_{\mathrm{F}} S_{3}^{\mathrm{T}}\left(n_{0}\right)$, where $S_{3}^{\mathrm{T}}=(3 / 4) S_{3}^{\mathrm{L}}$ and $S_{3}^{\mathrm{L}}$ is given by Eq. (16) of Ref. 61; (iii) at intermediate frequency, we require that $\operatorname{Im} \Delta K_{\mathrm{xc}}(\omega)$ and $\operatorname{Im} \Delta \mu_{\mathrm{xc}}(\mathbf{r}, \omega)$ have peaks around $\omega=2 \omega_{p}$ (where $\omega_{p}=\sqrt{4 \pi n_{0} e^{2} / m}$ is the plasmon frequency) as observed in the homogeneous electron gas; ${ }^{60,61}$ (iv) $K_{\mathrm{xc}}$ and $\mu_{\mathrm{xc}}$ must satisfy the sum rules (69) and (70). Among these conditions, only the last one takes the inhomogeneity parameters explicitly into account.

To satisfy the four conditions, we choose $\operatorname{Im} \Delta K_{\mathrm{xc}}(\mathbf{r}, \omega)$ and $\operatorname{Im} \Delta \mu_{\mathrm{xc}}(\mathbf{r}, \omega)$ to be of the simple form

$$
\begin{gathered}
\operatorname{Im} \Delta \mu_{\mathrm{xc}}=-\omega n_{0} f_{\mathrm{T}}(\mathbf{r}, \omega), \\
\operatorname{Im} \Delta K_{\mathrm{xc}}=-\omega n_{0} f_{\mathrm{L}}(\mathbf{r}, \omega)-\frac{4}{3} \operatorname{Im} \Delta \mu_{\mathrm{xc}},
\end{gathered}
$$

where

$$
\begin{gathered}
f_{\mathrm{L}(\mathrm{T})}=a_{\mathrm{L}(\mathrm{T})} /\left(1+b_{\mathrm{L}(\mathrm{T})} \widetilde{\omega}^{2}\right)^{5 / 4}+d_{\mathrm{L}(\mathrm{T})} \widetilde{\omega}^{2} e^{-\beta_{\mathrm{L}(\mathrm{T})} \widetilde{\omega}^{2}}, \\
a_{\mathrm{L}(\mathrm{T})}=n_{0}\left(m e^{2} / n_{0} \pi\right)^{2} k_{\mathrm{F}} S_{3}^{\mathrm{L}(\mathrm{T})}, \\
b_{\mathrm{L}(\mathrm{T})}=16\left[3 a_{\mathrm{L}(\mathrm{T})}^{4} /\left(4 r_{s}^{3} c_{\mathrm{L}(\mathrm{T})}^{4}\right)\right]^{1 / 5}, \\
\beta_{\mathrm{L}(\mathrm{T})}=3 / 2, \\
d_{\mathrm{L}(\mathrm{T})}=4 \sqrt{\beta_{\mathrm{L}(\mathrm{T})}^{3} / \pi} \\
\times\left[\frac{-\pi n_{0}}{4 \omega_{p}} \Delta_{\mathrm{L}(\mathrm{T})}-\frac{\pi \sqrt{2 \pi}}{2\left[\Gamma\left(\frac{1}{4}\right)\right]^{2}}\left(4 r_{s}^{3} c_{\mathrm{L}(\mathrm{T})}^{4} / 3\right)^{1 / 10} a_{\mathrm{L}(\mathrm{T})}^{3 / 5}\right],
\end{gathered}
$$

with $\widetilde{\omega}=\omega / \omega_{p}, \quad c_{\mathrm{L}}=23 / 15, \quad c_{\mathrm{T}}=16 / 15, \quad \Delta_{\mathrm{T}}=\left(\mu_{\mathrm{xc}}^{0}-\mu_{\mathrm{xc}}^{\infty}\right) / n_{0}^{2}$, and $\Delta_{\mathrm{L}}=\left(K_{\mathrm{xc}}^{0}-K_{\mathrm{xc}}^{\infty}\right) / n_{0}^{2}+4 \Delta_{\mathrm{T}} / 3$. Among the inputs $K_{\mathrm{xc}}^{\infty}, \mu_{\mathrm{xc}}^{\infty}$, $K_{\mathrm{xc}}^{0}$, and $\mu_{\mathrm{xc}}^{0}$, the last is the least accurately known. Even so, an estimate of this quantity for the uniform electron gas is available within the random phase approximation (RPA). ${ }^{60}$ The calculation beyond the RPA is still under study.

At the VK (LDA) level, the high- and low-frequency limits of the XC elastic moduli only depend upon the groundstate density. At the GGA level, while $K_{\mathrm{xc}}^{\infty}$ and $\mu_{\mathrm{xc}}^{\infty}$ additionally depend upon the first derivative of the density, $K_{\mathrm{xc}}^{0}$ depends not only on the density gradient, but also on the Laplacian of the density, as shown in the Appendix. To show the effect of the inhomogeneity on the elastic moduli, we calculate all these quantities within the GGA. ${ }^{33}$ For simplicity, we assume that the second derivative of the density is zero. The effect of the density gradient on the bulk and shear moduli is displayed in Figs. 1-3. From these figures, we see that the finite density gradient introduces significant corrections to the values of $K_{\mathrm{xc}}^{\mathrm{h}, 0}\left(n_{0}\right), K_{\mathrm{xc}}^{\mathrm{h}, \infty}\left(n_{0}\right)$, and $\mu_{\mathrm{xc}}^{\mathrm{h}, \infty}\left(n_{0}\right)$.

In continuum mechanics, the bulk and shear viscosities

$$
\zeta(\omega)=-\operatorname{Im} \Delta K_{\mathrm{xc}}(\mathbf{r}, \omega) / \omega
$$

and 


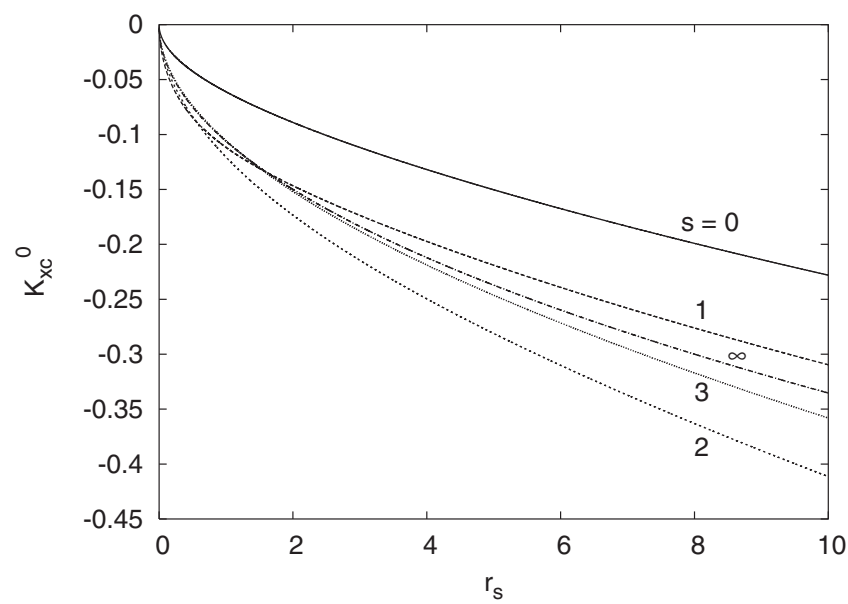

FIG. 1. The zero-frequency bulk modulus $K_{\mathrm{xc}}^{0}$ (in units of $\left.2 \omega_{p} n_{0}\right)$ as a function of $r_{s}$ for several values of the reduced gradient $s=\left|\nabla n_{0}\right| /\left[2\left(3 \pi^{2}\right)^{1 / 3}\right] . s=0$ corresponds to the uniform electron gas.

$$
\eta(\omega)=-\operatorname{Im} \Delta \mu_{\mathrm{xc}}(\omega) / \omega
$$

have direct physical significance. ${ }^{35}$ So we calculate these two quantities as functions of frequency by substituting $K_{\mathrm{xc}}^{\infty}, K_{\mathrm{xc}}^{0}$, and $\mu_{\mathrm{xc}}^{\infty}$ into Eq. (71) for several values of the reduced density gradient $s$ in the physical range of $0 \leqslant s \leqslant 3$ at a fixed density. As shown in Figs. 4 and 5, the viscosity spectra have an interesting evolution as functions of the density gradient. The viscosity at first increases with increasing density gradient. However, this trend is reversed in the large-gradient $(s \rightarrow \infty)$ limit. In this limit the exchange energy density of the GGA (and meta-GGA) takes the same form as in the uniform gas, but enhanced by a factor 1.804 , while the correlation part vanishes. ${ }^{33}$ Therefore the viscosity spectra tend in the $s \rightarrow \infty$ limit to $s$-independent functions, qualitatively similar to those of the homogeneous electron liquid.

Strong variations with $s$ are also found in the real parts of the bulk and shear moduli, which are calculated from Eqs. (67) and (68) and are shown in Figs. 6 and 7. From these

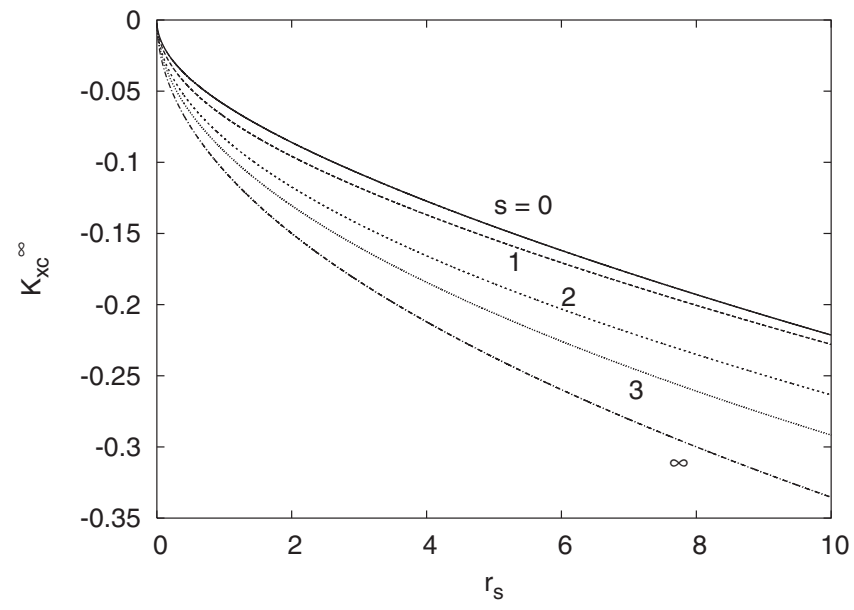

FIG. 2. The high-frequency bulk modulus $K_{\mathrm{xc}}^{\infty}$ (in units of $\left.2 \omega_{p} n_{0}\right)$ as a function of $r_{s}$ for several values of $s$.

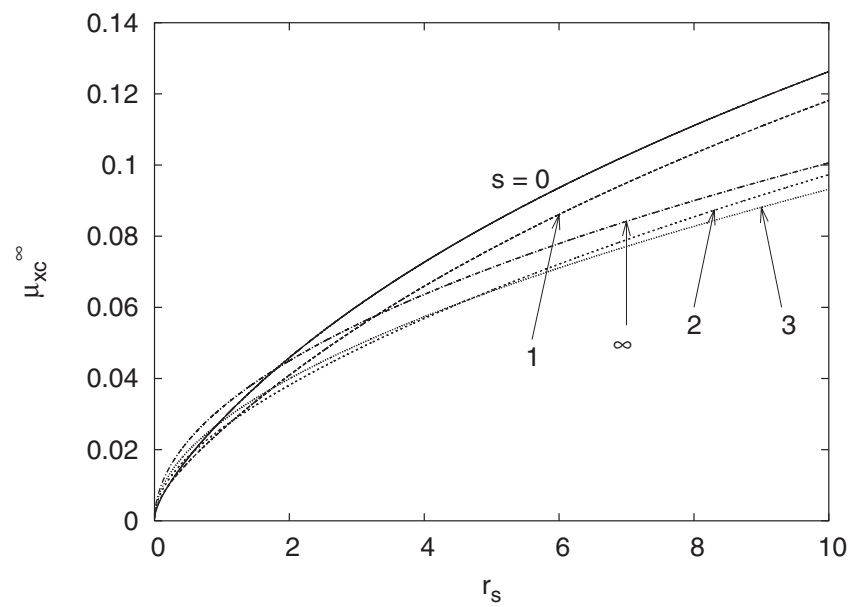

FIG. 3. The high-frequency shear modulus $\mu_{\mathrm{xc}}^{\infty}$ (in units of $\left.2 \omega_{p} n_{0}\right)$ as a function of $r_{s}$ for several values of $s$.

figures we see that in the range of $0 \leqslant s \lesssim 3$ the shear modulus $\mu_{\mathrm{xc}}$ of the inhomogeneous system is smaller than that of the homogeneous electron gas for most frequencies. These variations may lead to a better description of the optical properties of atoms, molecules, and molecular polymers. ${ }^{62}$

\section{CONCLUSION}

In conclusion, we have presented a detailed derivation of the recently proposed gradient-corrected frequencydependent XC potential. The proposed potential should allow a more accurate treatment of moderately to strongly inhomogeneous electronic systems (e.g., molecular junctions), for which the nonadiabatic local density approximation may not be adequate. At the same time the potential remains essentially exact for slowly varying densities and slowly varying time-dependent fields. Granted the smallness and slow variation of the strain $u_{\alpha \beta}(\mathbf{r})$, the quality of our approximation depends only on how well the GGA and the meta-GGA treat

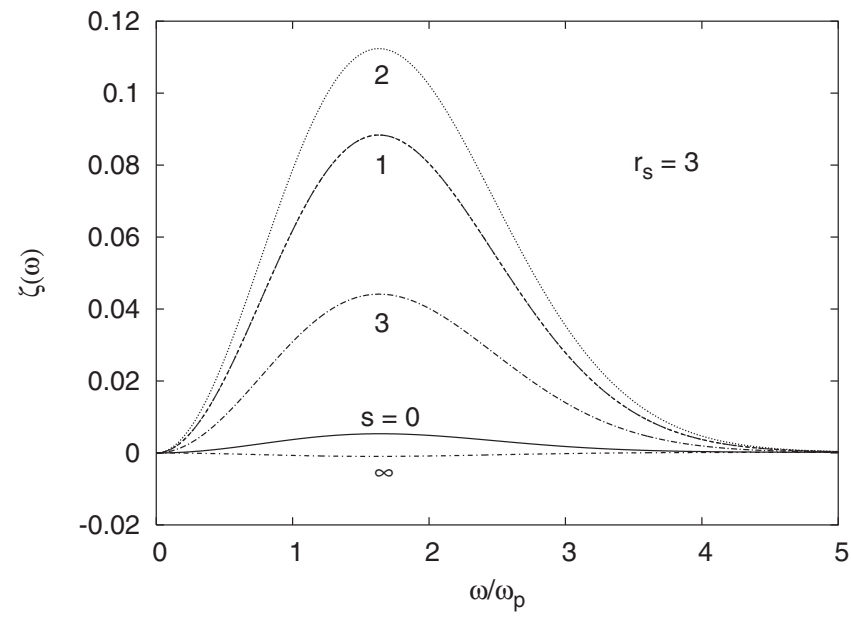

FIG. 4. The bulk viscosity $\zeta(\omega)$ (in units of $\hbar n_{0}$ ) for several values of $s$ at $r_{s}=3$. The same RPA value of $\mu_{\mathrm{xc}}(0)$ for all $s$ is taken from Table I of Ref. 60. 


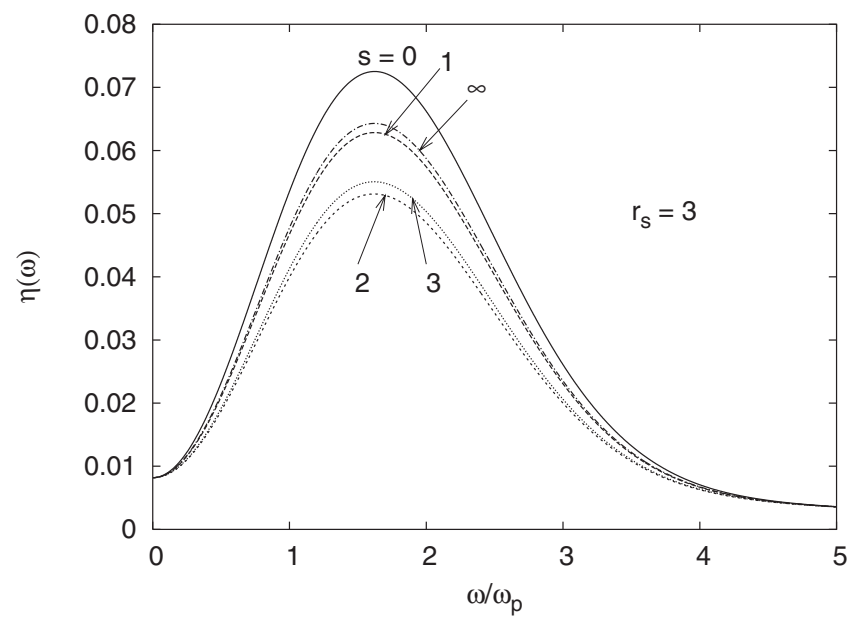

FIG. 5. The shear viscosity $\eta(\omega)$ (in units of $\hbar n_{0}$ ) for several values of $s$ at $r_{s}=3$. The same $\mu_{\mathrm{xc}}(0)$ for all $s$ is employed as in Fig. 4.

the static inhomogeneous system. As a simple example, we have employed the GGA functional to calculate the bulk modulus and the shear modulus of an electron gas with linear variation of the density. In practical applications, the dynamical potential based on the meta-GGA may be more accurate, ${ }^{63}$ in particular for finite systems.

The procedure employed to construct the nonadiabatic part of the XC field is summarized as follows. First we find the infinite-frequency limits $K_{\mathrm{xc}}^{\infty}$ and $\mu_{\mathrm{xc}}^{\infty}$ of the bulk and shear moduli, respectively [see Eq. (7)], by making use of semilocal density functionals to calculate the kinetic energy density of correlation (exchange does not contribute) and the $\mathrm{XC}$ potential energy density [see Eqs. (63) and (64)]. Combining these with the zero-frequency limits $K_{\mathrm{xc}}^{0}$ [Eq. (61)] and $\mu_{\mathrm{xc}}^{0}$, we calculate the frequency-dependent imaginary parts of the bulk and shear moduli by means of the interpolation formula (71). Their real parts are then obtained from the dispersion relations (67) and (68). Finally the dynamical $\mathrm{XC}$ stress tensor $\Delta P_{\mu \nu}(\mathbf{r}, \omega)$ is constructed from Eq. (3) by

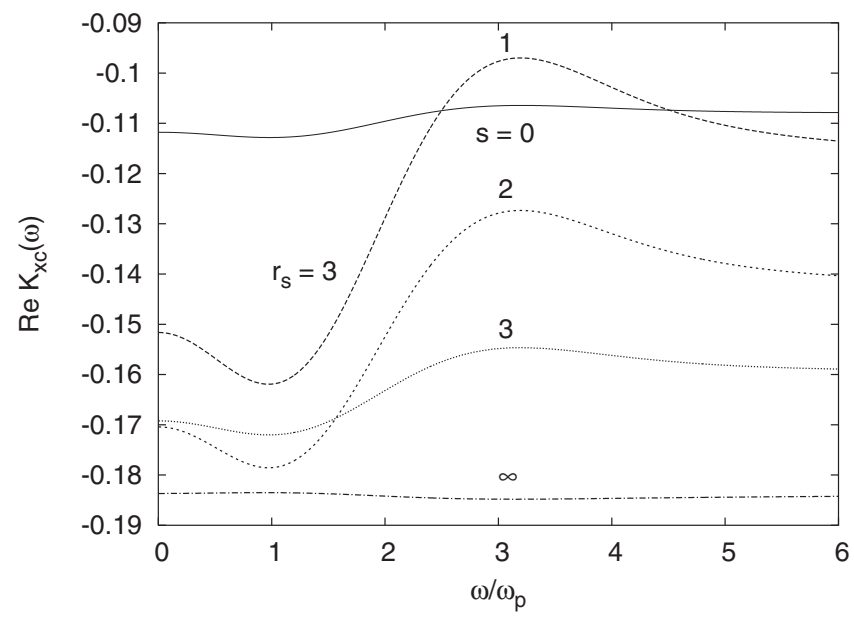

FIG. 6. The real part of the finite-frequency bulk modulus $\operatorname{Re} K_{\mathrm{xc}}(\omega)$ (in units of $2 \omega_{p} n_{0}$ ) for several values of $s$ at $r_{s}=3$.

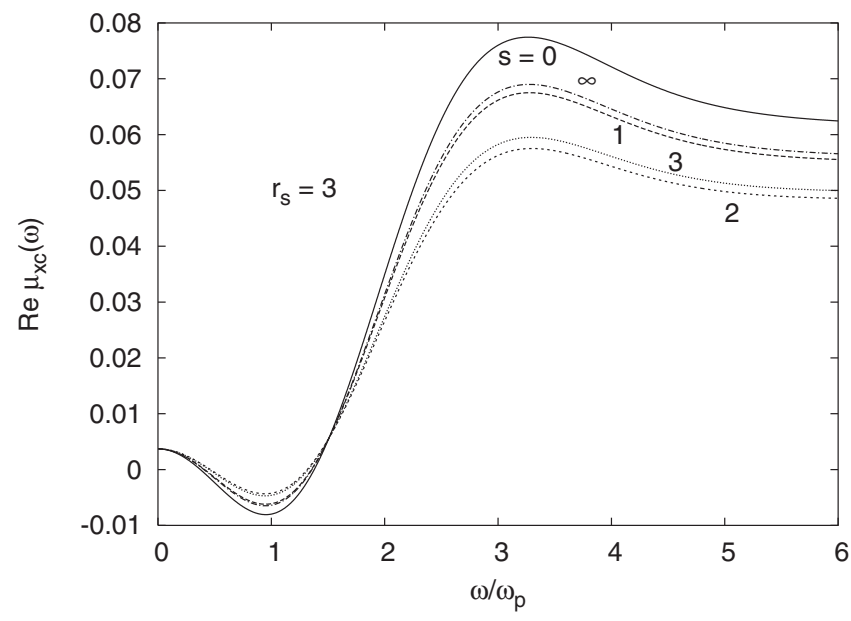

FIG. 7. The real part of the finite-frequency shear modulus $\operatorname{Re} \mu_{\mathrm{xc}}(\omega)$ (in units of $2 \omega_{p} n_{0}$ ) for several values of $s$ at $r_{s}=3$.

replacing $K_{\mathrm{xc}}^{\mathrm{h}}\left(n_{0}, \omega\right), \mu_{\mathrm{xc}}^{\mathrm{h}}\left(n_{0}, \omega\right)$, and $K_{\mathrm{xc}}^{\mathrm{h}, 0}\left(n_{0}\right)$ with the corresponding parts for the inhomogeneous system.

\section{ACKNOWLEDGMENT}

This work was supported by the DOE under Grant No. DE-FG02-05ER46203.

\section{APPENDIX: LOW-FREQUENCY LIMIT OF THE BULK MODULUS}

Self-consistent implementations of time-dependent DFT require the dynamical XC potential, the divergence of the stress tensor, which may be calculated from the bulk and shear moduli.

At the GGA level, $P^{\mathrm{xc}, \mathrm{eq}}$ is an explicit functional of the ground-state density $n_{0}$. The functional derivative of Eq. (61) can be written as

$$
\begin{aligned}
\frac{\delta P^{\mathrm{xc}}\left(\left[n_{0}\right] ; \mathbf{r}\right)}{\delta n_{0}\left(\mathbf{r}^{\prime}\right)}= & {\left[\frac{\partial P^{\mathrm{xc}}\left(\left[n_{0}\right] ; \mathbf{r}\right)}{\partial n_{0}(\mathbf{r})}-\nabla \cdot \frac{\partial P^{\mathrm{xc}}\left(\left[n_{0}\right] ; \mathbf{r}\right)}{\partial \boldsymbol{\nabla} n_{0}(\mathbf{r})}\right] } \\
& \times \delta\left(\mathbf{r}-\mathbf{r}^{\prime}\right) .
\end{aligned}
$$

Applying Eq. (A1) to the GGA functional of Ref. 33, we find the low-frequency limit of the bulk modulus,

$$
\begin{aligned}
K_{\mathrm{xc}}^{0}= & \frac{n_{0}}{3} v_{x}^{\mathrm{PBE}}-\frac{1}{6 \pi r_{s}^{2}} \frac{\partial \epsilon_{\mathrm{c}}^{\mathrm{unif}}}{\partial r_{s}}+\frac{1}{12 \pi r_{s}} \frac{\partial^{2} \epsilon_{\mathrm{c}}^{\mathrm{unif}}}{\partial r_{s}^{2}}-\frac{1}{6 \pi r_{s}^{2}} G \\
& -\frac{n_{0}^{2} r_{s}}{3} \frac{\partial G}{\partial n_{0}}+\frac{n_{0}}{3} \boldsymbol{\nabla} \cdot\left(n_{0} r_{s} \frac{\partial G}{\partial \boldsymbol{\nabla} n_{0}}\right)
\end{aligned}
$$

where

$$
\frac{\partial \epsilon_{\mathrm{c}}^{\mathrm{unif}}}{\partial r_{s}}=-2 c_{0} \alpha_{1} \ln \left(1+\frac{1}{Q_{1}}\right)-\frac{Q_{0} Q_{1}^{\prime}}{Q_{1}+Q_{1}^{2}},
$$




$$
\frac{\partial^{2} \epsilon_{\mathrm{c}}^{\text {unif }}}{\partial r_{s}^{2}}=\frac{1}{Q_{1}+Q_{1}^{2}}\left[4 c_{0} \alpha_{1} Q_{1}^{\prime}-Q_{0} Q_{1}^{\prime \prime}+\frac{Q_{0}\left(1+2 Q_{1}\right) Q_{1}^{\prime 2}}{Q_{1}+Q_{1}^{2}}\right]
$$

are the local contributions. Here $Q_{0}=-2 c_{0}\left(1+\alpha_{1} r_{s}\right)$, $Q_{1}=2 c_{0}\left(\beta_{1} r_{s}^{1 / 2}+\beta_{2} r_{s}+\beta_{3} r_{s}^{3 / 2}+\beta_{4} r_{s}^{p+1}, Q_{1}^{\prime}=c_{0}\left[\beta_{1} r_{s}^{-1 / 2}+2 \beta_{2}\right.\right.$ $\left.\left.+3 \beta_{3} r_{s}^{1 / 2}+2(p+1) \beta_{4} r_{s}^{p}\right)\right]$, and $Q_{1}^{\prime \prime}=\left(c_{0} / 2\right)\left[-\beta_{1} r_{s}^{-3 / 2}+3 \beta_{3} r_{s}^{-1 / 2}\right.$ $\left.+4 p(p+1) r_{s}^{p-1}\right]$, and all the parameters are given in Table I of Ref. 64. The nonlocal terms are calculated from

$$
G=-\frac{\beta \phi^{3} t^{2}}{\left(1+\beta B t^{2} / \gamma\right)}\left[\frac{B}{r_{s}}+\frac{A t^{4}}{D^{2}}\left(2+A t^{2}\right)\left(\frac{\partial A}{\partial r_{s}}-\frac{A}{r_{s}}\right)\right]
$$

$$
\begin{aligned}
\frac{\partial G}{\partial \boldsymbol{\nabla} n_{0}}= & \frac{\beta \phi \boldsymbol{\nabla} n_{0}}{16(4)^{1 / 3} r_{s} n_{0}^{8 / 3}}\left\{\frac { 1 } { 1 + \beta B t ^ { 2 } / \gamma } \left[-2 \frac{B}{r_{s}}+\frac{2 A^{2} t^{4}}{r_{s} D^{2}}\left(2+A t^{2}\right)-\frac{2 A t^{4}}{D^{2}}\left(4+3 A t^{2}\right)\left(\frac{\partial A}{\partial r_{s}}-\frac{A}{r_{s}}\right)\right.\right. \\
& \left.\left.+\frac{4 A^{2} t^{6}}{D^{3}}\left(2+A t^{2}\right)\left(1+2 A t^{2}\right)\left(\frac{\partial A}{\partial r_{s}}-\frac{A}{r_{s}}\right)\right]-\frac{2 \beta t^{2}}{\gamma\left(1+\beta B t^{2} / \gamma\right)^{2} D^{2}}\left[-\frac{B}{r_{s}}-\frac{A t^{4}}{D^{2}}\left(2+A t^{2}\right)\left(\frac{\partial A}{\partial r_{s}}-\frac{A}{r_{s}}\right)\right]\left(1+2 A t^{2}\right)\right\} .
\end{aligned}
$$

In Eqs. (A5)-(A7), $t=(c s / \phi) / r_{s}^{1 / 2}$, where $c=\left(3 \pi^{2} / 16\right)^{1 / 3}$ and $s$ is the reduced density gradient defined in the context, $\phi=[(1$ $\left.+\zeta)^{2 / 3}+(1-\zeta)^{2 / 3}\right] / 2, \quad \gamma=0.031091, \quad \beta=0.066725, \quad E=\exp \left[-\epsilon_{\mathrm{c}}^{\text {unif }} /\left(\gamma \phi^{3}\right)\right], \quad A=\beta /[\gamma(E-1)], \quad \partial A / \partial r_{s}=\beta E\left(\partial \epsilon_{\mathrm{c}}^{\text {unif }} / \partial r_{s}\right) /\left[\gamma^{2} \phi^{3}(E\right.$ $\left.-1)^{2}\right], B=\left(1+A t^{2}\right) / D$, and $D=1+A t^{2}+A^{2} t^{4}$. In the uniform-gas limit, all the $G$ terms vanish and we are left with the local density approximation for the moduli.

*Present address: Theoretical Division, Los Alamos National Laboratory, Los Alamos, NM 87545.

†jtao@lanl.gov

${ }^{1}$ E. Runge and E. K. U. Gross, Phys. Rev. Lett. 52, 997 (1984).

${ }^{2}$ G. F. Giuliani and G. Vignale, Quantum Theory of the Electron Liquid (Cambridge University Press, Cambridge, England, 2005).

${ }^{3}$ A. Zangwill and P. Soven, Phys. Rev. Lett. 45, 204 (1980); Phys. Rev. A 21, 1561 (1980).

${ }^{4}$ R. Bauernschmitt and R. Ahlrichs, Chem. Phys. Lett. 256, 454 (1996).

${ }^{5}$ D. Jacquemin, M. Bouhy, and E. A. Perpète, J. Chem. Phys. 124, 204321 (2006)

${ }^{6}$ S. J. A. van Gisbergen, J. G. Snijders, and E. J. Baerends, J. Chem. Phys. 103, 9347 (1995).

${ }^{7}$ R. D'Agosta and G. Vignale, Phys. Rev. Lett. 96, 016405 (2006).

${ }^{8}$ C. A. Ullirich, J. Chem. Phys. 125, 234108 (2006).

${ }^{9}$ D. J. Tozer and N. C. Handy, Phys. Chem. Chem. Phys. 2, 2117 (2000).

${ }^{10}$ D. J. Tozer, R. D. Amos, N. C. Handy, B. O. Roos, and L. Serrano-Andres, Mol. Phys. 97, 859 (1999).

${ }^{11}$ A. Dreuw, J. L. Weisman, and M. Head-Gordan, J. Chem. Phys.
119, 2943 (2003).

${ }^{12}$ D. J. Tozer, J. Chem. Phys. 119, 12697 (2003).

${ }^{13}$ N. T. Maitra, J. Chem. Phys. 122, 234104 (2005).

${ }^{14}$ W. Hieringer and A. Gorling, Chem. Phys. Lett. 419, 557 (2006).

${ }^{15}$ M. van Faassen, P. L. de Boeij, R. van Leeuwen, J. A. Berger, and J. G. Snijders, Phys. Rev. Lett. 88, 186401 (2002).

${ }^{16}$ M. van Faassen, P. L. de Boeij, R. van Leeuwen, J. A. Berger, and J. G. Snijders, J. Chem. Phys. 118, 1044 (2003).

${ }^{17}$ W. G. Aulbur, L. W. Jonsson, and J. W. Wilkins, in Solid State Physics, edited by H. Ehrenfeich and F. Spaepeu (Academic Press, New York, 2000), p. 1.

${ }^{18}$ J. Tao and G. Vignale, Phys. Rev. Lett. 97, 036403 (2006). This statement is misprinted as ALDA severely underestimates the dielectric polarizability of insulators. The word "underestimates" should be corrected as "overestimates."

${ }^{19}$ E. S. Kadantsev, M. J. Stott, and A. Rubio, J. Chem. Phys. 124, 134901 (2006).

${ }^{20}$ E. K. U. Gross and W. Kohn, Phys. Rev. Lett. 55, 2850 (1985).

${ }^{21}$ J. F. Dobson, Phys. Rev. Lett. 73, 2244 (1994).

${ }^{22}$ G. Vignale, Phys. Rev. Lett. 74, 3233 (1995).

${ }^{23}$ G. Vignale and W. Kohn, Phys. Rev. Lett. 77, 2037 (1996); in Electronic Density Functional Theory, edited by J. Dobson, M. 
P. Das, and G. Vignale (Plenum Press, New York, 1998).

${ }^{24}$ G. Vignale, C. A. Ullrich, and S. Conti, Phys. Rev. Lett. 79, 4878 (1997).

${ }^{25}$ J. A. Berger, P. L. de Boeij, and R. van Leeuwen, Phys. Rev. B 75, 035116 (2007).

${ }^{26}$ C. A. Ullrich and G. Vignale, Phys. Rev. Lett. 87, 037402 (2001).

${ }^{27}$ M. van Faassen and P. L. de Boeij, J. Chem. Phys. 120, 8353 (2004).

${ }^{28}$ C. A. Ullrich and K. Burke, J. Chem. Phys. 121, 28 (2004).

${ }^{29}$ M. K. Harbola and V. Sahni, Phys. Rev. Lett. 62, 489 (1989).

${ }^{30}$ Y. Wang, J. P. Perdew, J. A. Chevary, L. D. Macdonald, and S. H. Vosko, Phys. Rev. A 41, 78 (1990).

${ }^{31}$ A. Holas and N. H. March, Phys. Rev. A 51, 2040 (1995).

${ }^{32}$ A. D. Becke, Phys. Rev. A 38, 3098 (1988); C. Lee, W. Yang, and R. G. Parr, Phys. Rev. B 37, 785 (1988).

${ }^{33}$ J. P. Perdew, K. Burke, and M. Ernzerhof, Phys. Rev. Lett. 77, 3865 (1996).

${ }^{34}$ J. Tao, J. P. Perdew, V. N. Staroverov, and G. E. Scuseria, Phys. Rev. Lett. 91, 146401 (2003).

${ }^{35} \mathrm{Na}$ Sai, M. Zwolak, G. Vignale, and M. Di Ventra, Phys. Rev. Lett. 94, 186810 (2005).

${ }^{36}$ I. V. Tokatly, Phys. Rev. B 71, 165104 (2005); 71, 165105 (2005).

${ }^{37}$ I. V. Tokatly, Lect. Notes Phys. 706, 123 (2006).

${ }^{38}$ C. A. Ullrich and I. V. Tokatly, Phys. Rev. B 73, 235102 (2006).

${ }^{39}$ I. V. Tokatly, Phys. Rev. B 75, 125105 (2007).

${ }^{40}$ C. L. Rogers and A. M. Rappe, Phys. Rev. B 65, 224117 (2002).

${ }^{41}$ D. Pines and P. Nozières, The Theory of Quantum Liquids (Benjamin, New York, 1966), Vol. 1.

${ }^{42}$ G. B. Arfken and H. J. Weber, Mathematical Methods for Physicists (Academic Press, New York, 1985).

${ }^{43}$ A. D. Becke, Int. J. Quantum Chem. 23, 1915 (1983).

${ }^{44}$ J. P. Perdew, K. Burke, and Y. Wang, Phys. Rev. B 54, 16533 (1996).

${ }^{45}$ L. A. Constantin, J. P. Perdew, and J. Tao, Phys. Rev. B 73,
205104 (2006).

${ }^{46}$ A. D. Becke and M. R. Roussel, Phys. Rev. A 39, 3761 (1989).

${ }^{47}$ J. P. Perdew, A. Ruzsinszky, J. Tao, V. N. Staroverov, G. E. Scuseria, and G. I. Csonka, J. Chem. Phys. 123, 062201 (2005).

${ }^{48}$ V. N. Staroverov, G. E. Scuseria, J. Tao, and J. P. Perdew, Phys. Rev. B 69, 075102 (2004).

${ }^{49}$ S. Conti and G. Vignale, Phys. Rev. B 60, 7966 (1999).

${ }^{50}$ D. C. Langreth and J. P. Perdew, Solid State Commun. 17, 1425 (1975).

${ }^{51}$ O. Gunnarsson and B. I. Lundqvist, Phys. Rev. B 13, 4274 (1976).

${ }^{52}$ A. Görling, M. Levy, and J. P. Perdew, Phys. Rev. B 47, 1167 (1993).

${ }^{53}$ J. Tao and J. P. Perdew, in Reviews in Modern Quantum Chemistry: A Celebration of the Contributions of R. G. Parr, edited by K. D. Sen (World Scientific, Singapore, 2002).

${ }^{54}$ V. N. Staroverov, G. E. Scuseria, J. Tao, and J. P. Perdew, J. Chem. Phys. 119, 12129 (2003); 121, 11507(E) (2004).

${ }^{55}$ F. Furche and J. P. Perdew, J. Chem. Phys. 124, 044103 (2006).

${ }^{56}$ J. Tao, Phys. Rev. B 71, 205107 (2005). The coefficient of $1 / 72$ of the density gradient term of the kinetic energy density $\tilde{\tau}_{\sigma}$ below Eq. (25) is misprinted as $1 / 8$.

${ }^{57}$ J. Tao and J. P. Perdew, Phys. Rev. Lett. 95, 196403 (2005).

${ }^{58}$ P. Jurecka P, J. Cerny, P. Hobza, and D. R. Salahub, J. Comput. Chem. 28, 555 (2007).

${ }^{59}$ A. J. Glick and W. F. Long, Phys. Rev. B 4, 3455 (1971).

${ }^{60}$ R. Nifosì, S. Conti, and M. P. Tosi, Phys. Rev. B 58, 12758 (1998).

${ }^{61}$ Z. Qian and G. Vignale, Phys. Rev. B 65, 235121 (2002).

${ }^{62}$ J. A. Berger, P. L. de Boeij, and R. van Leeuwen, Phys. Rev. B 71, 155104 (2005).

${ }^{63}$ J. P. Perdew, J. Tao, V. N. Staroverov, and G. E. Scuseria, J. Chem. Phys. 120, 6898 (2004).

${ }^{64}$ J. P. Perdew and Y. Wang, Phys. Rev. B 45, 13244 (1992). 